\title{
Los megaeventos: indicadores del archipiélago urbano mundial desde una perspectiva cultural basada en el consumo de eventos de ocio ${ }^{1}$
}

\author{
Roberto D. Pisonero. Universidad Complutense de Madrid, Madrid, España; \\ ILs-Research Institute for Regional and Urban Development, Dortmund, Alemania.
}

RESUMEN | En la actualidad, las ciudades no compiten únicamente para atraer flujos económicos, sino también por formas particulares de capital cultural (símbolos, imágenes, experiencias) que enfaticen su identidad y su valor cosmopolita. En este sentido, los eventos y festivales culturales se han convertido no solo en una estrategia de citymarketing inigualable, sino que, además, pueden utilizarse como indicadores estadísticos para evaluar el archipiélago mundial de ciudades desde una perspectiva cultural contextualizada en la actual sociedad de ocio-consumo. Siguiendo una metodología cuantitativa que incluye varios análisis estadísticos, los resultados corroboran dos hechos significativos: por un lado, la necesidad de estudiar el sistema urbano mundial desde una perspectiva multidimensional, más allá de criterios económicos; por otro, lo importante de detenerse no solo en las denominadas ciudades globales, identificadas ampliamente en la literatura científica, sino también en aquellas secundarias que, gracias a los acontecimientos vinculados al ocio y la cultura, se posicionan en el escenario global.

PALABRAS CLAVE | sistema urbano, globalización, marketing urbano.

ABSTRACT | In the present, cities do not only compete to attract economic flows, but also particular forms of cultural capital (symbols, images, experiences) that emphasize the identity and cosmopolitan value of these. Events and cultural festivals that are often unique city-marketing strategies can also be used as indicators in statistical analyses to assess the global urban system from the cultural perspective that is contextualized in today's consumer society. Following a quantitative methodology that includes various statistical analyses, the results confirm two important facts: on the one hand, the need to study the global urban system from a multidimensional perspective, beyond economic criteria; on the other hand, the need to observe not only the so-called global cities, widely identified in the scientific literature, but also in those secondary cities that are positioned on the global stage through these events. KEYWORDS | urban system, globalization, urban marketing.

1 Este artículo se enmarca en el proyecto de investigación competitivo "Lugares, imaginarios, y movilidades turísticas en tiempos de crisis", CSO2OII-26527, financiado por el Ministerio de Ciencia e Innovación del Gobierno de España. 


\section{Introducción}

Las llamadas "sociedad del ocio" y "sociedad del consumo" (Bauman, 2007; Lash \& Urry, 1994; Lipovetsky, 2003, 2006; Ritzer, 2006) o "hiperconsumo" (Lipovetsky \& Serroy, 2015) se han convertido en dos de las manifestaciones más destacadas de la globalización. Ambos elementos, ocio y consumo, se han difundido y generalizado en las ciudades, especialmente en aquellos núcleos jerárquicos superiores considerados los máximos exponentes de las prácticas de producción y consumo cultural (Florida, 2002, 2005; Scott, 2009, 2010), hecho que favorece el desarrollo de la globalización cultural.

En este sentido, la hipótesis de este trabajo considera la necesidad de incorporar la dimensión cultural (a través de los grandes eventos y festivales culturales) en el conocimiento del sistema urbano mundial desde una perspectiva multidimensional y, a nuestro juicio, más adaptada a las formas y consumos culturales actuales, enmarcados en el denominado capitalismo cultural cognitivo (Scott, 2008). Solo de esta manera se evitan aproximaciones sectoriales que ofrecen un panorama sesgado de la realidad, como ocurre tradicionalmente en rankings urbanos de corte economicista.

La hipótesis también sostiene que las investigaciones en temas urbanos no debieran centrarse exclusivamente en las ciudades más representativas de la jerarquía urbana mundial, sino también en los flujos y conexiones territoriales del resto de ciudades, utilizando para ello una perspectiva multiescalar. Siguiendo la idea de Marcuse y Van Kempen (2000), que consideran la globalización como un proceso que se manifiesta en diferentes formas e intensidades, se pretende no solo identificar aquellas ciudades consideradas globales, sino también valorar aquellas otras donde determinadas especificidades establecen un hinterland, también de amplio alcance, aunque mucho más especializado desde el punto de vista funcional. Estas ciudades, aunque un poco alejadas conceptualmente de la definición de ciudad mundial aceptada en el mundo académico, también contribuyen a la organización del territorio.

Para lograr el objetivo señalado, se utilizarán los eventos y festivales internacionales como indicadores de orden cultural, interpretados bajo parámetros que reflejan una cultura de masas propia de la sociedad de ocio-consumo actual. El objetivo de esta investigación no consiste en analizar los impactos económicos, sociales, políticos y/o urbanos de estos acontecimientos en las ciudades anfitrionas a modo de estudios de caso, puesto que existe una dilatada literatura que acomete tal fin (Waitt, 2003; Herrero, Sanz, Devesa, Begate \& Del Barrio, 2006; Kim, Gursoy \& Lee, 2006; Gaffney, 2010; entre otros). Se considera, sin embargo, que menos trabajos se detienen en el conocimiento de dichos eventos desde una perspectiva holística, entendiéndolos no solo como estrategias de branding y citymarketing que fomentan la competitividad urbana y la proyección internacional de las ciudades (Evans, 2009; Maenning \& Zimbalist, 2012), sino también como indicadores de éxito que permiten determinar el sistema urbano mundial desde una perspectiva cultural. $^{2}$

2 Esta investigación, de índole geográfica, no pretende abordar el concepto de cultura desde perspectivas correspondientes a la antropología y la sociología, en donde ella constituye el contexto y la esencia de toda investigación. Así, reconociendo de antemano la imposibilidad de 
Son varios los objetivos que se persiguen en esta investigación: (i) proponer un indicador sintético de megaeventos (ISMeg), para demostrar cómo los mismos desempeñan un papel primordial en la actual organización territorial derivada de la globalización cultural; (ii) detectar las distintas estrategias y/o trayectorias funcionales generales (económicas y/o culturales) que desarrollan las ciudades del sistema urbano mundial, para lo cual se compararán dos rankings, uno de ellos confeccionado en este trabajo (objetivo 1) y otro de temática fundamentalmente económica, elaborado por el grupo de Investigación Globalization and World Cities (Gawc) de la Universidad de Loughborough (Reino Unido); (iii) identificar, por grandes conjuntos de ciudades, la organización del sistema urbano mundial, que se asume es de carácter complejo; el objetivo, en este caso, no es solo determinar las principales ciudades, sino aquellas otras que experimentan, con respecto a las primeras, procesos paralelos de globalización, aunque de componente más especializada; (iv) identificadas las estrategias generales, se pretende determinar más específicamente las estrategias funcionales de carácter cultural a partir de los eventos y festivales que las diferencian, con el fin de definir las estrategias relativas a la promoción de eventos (event strategies) que llevan a cabo los planificadores y dirigentes urbanos.

El trabajo, después de esta introducción, se organiza de la siguiente manera: en primer lugar, se expone el significado de los eventos deportivos y/o culturales en el contexto actual de la "economía cultural" dominada por el ocio y consumo de masas; a continuación, se presenta de manera concisa la metodología empleada en este trabajo; posteriormente, se exponen los resultados de la investigación; finalmente, se recopilan las conclusiones más significativas.

\section{Hacia una nueva competitividad basada en el factor cultural: el papel de los eventos}

Tradicionalmente, se ha dado un mayor peso específico a la cuestión económica en los trabajos sobre globalización. Sin embargo, este es un proceso mucho más complejo, donde tienen cabida multitud de manifestaciones que obligan a realizar estudios más holísticos y menos sectoriales, que no supediten ninguna de las áreas que puedan influir en el análisis del funcionamiento real de las dinámicas contemporáneas. En particular importa la dimensión cultural de las ciudades ante la notable convergencia entre el ámbito económico y cultural, denominada en la literatura especializada como "nueva economía” o "economía cultural" (Amin \& Thrift, 2007; Stoper \& Scott, 2009). Esta "nueva economía”, contextualizada en la actual sociedad del ocio y consumo (Bauman, 2007; Lash \& Urry, 1994; Ritzer, 2006), constituye una de las particularidades de los procesos contemporáneos de urbanización.

En este contexto, en tanto que forma de movilidad humana, el turismo se ha consolidado no solo como uno de los motores de la globalización, sino una de las mejores expresiones del consumo del ocio en la actualidad (Mansvelt, 2008;

medir el fenómeno cultural, por su naturaleza inmaterial y por la propia definición y esencia de lo que desde un punto de vista antropológico y sociológico puede considerarse como cultura, se propone una aproximación empírica, basada en eventos y festivales, destinados fundamentalmente al consumo de masas y cuya ubicación preferente es el espacio urbano. 
Urry, 1990). Hoy en día, este sector es considerado una de las "actividades más importantes del mundo, tanto por la movilidad de personas como por el volumen de negocio que genera" (Córdoba et al., 2010). Su carácter transversal implica que en su desarrollo se encuentran involucradas muchas actividades de índole cultural, como la recreación, los deportes, las artes, la música y los espectáculos, entre otras. En la mayoría de estas prácticas se pueden observar algunos de los valores predominantes en la nueva cultura posmoderna, como la búsqueda del placer, la diversión, las emociones o la estética (Canclini, 1995; Lipovetsky, 2007).

Desde la perspectiva señalada, las actividades y servicios culturales adquieren un especial significado en el archipiélago urbano mundial, pues hoy en día los centros urbanos son los máximos exponentes de las prácticas de producción y consumo cultural y, por consiguiente, elementos que propician el desarrollo de la globalización cultural. Ante la mutua dependencia que existe entre este tipo de actividades y los lugares en los que se implementan, muchas ciudades hacen hincapié en su oferta cultural con el fin de promover un mejor posicionamiento en la jerarquía mundial de centros atractivos. De esta forma, los servicios culturales pasan a ser el corazón de la actividad urbana (Currid, 2006; Scott, 2010) y los elementos fundamentales de competitividad entre ciudades (Méndez \& Sánchez, 2011). Es así que la creciente notoriedad de la dimensión creativa en las ciudades ha animado a los planificadores urbanos a pensar no solo en términos de localización de manufacturas y condiciones de producción competitivas (suelo, mano de obra y energía barata, buenas comunicaciones, etcétera), sino también a considerar las manifestaciones culturales de orden global; entre ellas, las actividades de ocio y esparcimiento, la dinámica creativa e innovadora, o la celebración de eventos y/o festivales culturales. ${ }^{3}$

Para algunos autores, estas manifestaciones se han convertido en vehículos indispensables no solo para el desarrollo socioeconómico (estimular el crecimiento económico, diversificar el producto turístico, impulsar la cohesión social), sino para la propia organización y desarrollo de todo el concepto de lo urbano (trama o plano, infraestructuras y fortalecimiento de la imagen, por ejemplo), facilitando la inclusión de las ciudades en la lógica de las industrias culturales (Power \& Nielsen, 2010). Las actividades y servicios culturales, por consiguiente, adquieren gran notoriedad, no solo como potencial heredado, sino como un valor creativo que permite proyectar nuevos factores de diferenciación urbana (Scott, 2010). Esto se considera especialmente relevante ante la actual competencia entre ciudades a escala global, que tratan no solo de crear una identidad que fomente su valor de marca, sino también distinguirse del resto en un contexto de homogeneización global.

3 Conviene matizar estas consideraciones, ya que el enfoque de la temática que se está tratando puede cuestionarse en lo referente a la competitividad como rasgo de muchas ciudades del mundo en desarrollo. Cierto es que la competitividad no solo se restringe al mundo desarrollado, sino que muchas ciudades del sur comienzan a posicionarse en el escenario competitivo global. Sin embargo, los motivos que justifican su creciente competitividad contrastan con los del norte. Nos referimos, por ejemplo, a las condiciones salariales (mucho más reducidas) o medioambientales (en ocasiones, sin ningún tipo de legislación al respecto), que pueden darles mayor atractivo. Para un estudio más detallado sobre la "Otra" competitividad, véase Chan y Ross (2002) o Shatking (2007), entre otros. 
Aunque no existe una "fórmula mágica" para crear una exitosa imagen de marca urbana (Evans, 2009), concurren diferentes orientaciones estratégicas para construir una identidad que fomente el valor de marca de una ciudad, su valor intangible, su valor inmaterial (Precedo, Orosa \& Míguez, 2010). Además de los logotipos y/o eslóganes, los edificios emblemáticos asociados a arquitectos "estrella” y los barrios urbanos donde se dan cita las tendencias y personajes de vanguardia (in o cool) en temas de moda y/o arte, se considera de sumo interés un gran compendio de iniciativas que, aunque están más vinculadas a cuestiones geopolíticas y no forman parte estricta del citymarketing, son utilizadas por este como una potente vía de promoción de la ciudad. Nos referimos a la celebración de eventos (culturales, deportivos, etcétera) y la creciente "festivalización" de algunas ciudades (Richards, 2007), proceso que las lleva a ser comúnmente conocidas en la literatura académica como "eventful cities", y que contribuye a la progresiva tematización de las mismas (Tressera, 2004). Estas iniciativas incluyen la celebración de eventos internacionales, tales como las Exposiciones Universales, los Juegos Olímpicos o las Copas del Mundo de fútbol, entre otros.

Existe actualmente una competencia creciente por albergar este tipo de actividades, especialmente entre aquellas ciudades consideradas de primer nivel, que "luchan" por escalar y alzarse con las primeras posiciones de la jerarquía urbana mundial. Respecto de estas ciudades premium se supone la existencia de importantes efectos acumulativos, por su aprovechamiento de economías de escala, condiciones extraordinarias de accesibilidad, el capital humano que en ellas reside, o sus importantes volúmenes de población, especialmente las denominadas 'clases creativas' (Florida, 2002, 2005), que son las que consumen en mayor grado la cultura.

El objetivo de "poner las ciudades en el mapa" se adapta a la escala y posibilidades de cada una, yendo desde megaeventos internacionales hasta ferias más modestas de carácter regional. Generalmente, las ciudades de mayor influencia internacional compiten por ser anfitrionas de los Juegos Olímpicos, Campeonatos Mundiales de diferentes deportes ${ }^{4}$ o Exposiciones Universales, entre otros acontecimientos. Estos constituyen los megaeventos culturales públicos más visibles y espectaculares de la sociedad actual (Getz, 2008) y, dada su repercusión y la importante inversión que se realiza antes de su celebración, son potentes impulsores de regeneración urbana. Las ciudades más pequeñas, por su parte, no quieren ser excluidas de este juego de intereses. Si no pueden competir por atraer este tipo de acontecimientos, juegan sus cartas desarrollando ferias de arte más modestas o festivales de cine (Bradley \& Hall, 2006).

Los eventos internacionales, en general, vienen definidos por su carácter localizado en el tiempo, con un importante esfuerzo presupuestario para su realización, un carácter mediático de las actividades planificadas y un umbral de población elevado para que la actividad pueda realizarse con éxito (Maenning \& Zimbalist, 2012). Existe una dilatada literatura académica acerca del impacto positivo que estas estrategias culturales tienen tanto en la economía local como en la imagen territorial de las ciudades anfitrionas, especialmente referente a los Juegos Olímpicos (Davis, 
2012; Essex \& Chalkley, 2003) y las Exposiciones Universales (Groote, 2005; Bou, 2009), simplemente por mencionar dos de las estrategias de mayor impacto internacional. Estos eventos son capaces de estimular las enormes inversiones que necesitan las ciudades para emprender reestructuraciones urbanas sin precedentes, corrigiendo déficits en las infraestructuras de turismo, transporte y/o alojamiento, a fin de garantizar la perfecta ejecución de los mismos (Essex \& Chalkley, 2003). También es cierto que en muchos casos sus impactos son ampliamente cuestionados por quienes los consideran burdos desarrollos de un urbanismo neoliberal, que supone una importante gentrificación de ciertas zonas de las urbes y el desplazamiento de grupos sociales menos desfavorecidos (Gaffney, 2010). ${ }^{5}$

No obstante lo anterior, lo que se antoja más relevante en la dimensión global es la repercusión de los grandes eventos en la imagen de las ciudades. Estos megaproyectos urbanos no solo transforman y modernizan el tejido urbano, sino que contribuyen a la diversificación del producto turístico y mejoran la imagen del lugar que, al menos durante unos días, se convierte en la capital cultural del mundo (Maenning \& Zimbalist, 2012). El gran número de países participantes, de turistas que asisten y de telespectadores que los siguen en todo el planeta convierten a los grandes eventos deportivos y/o culturales en acontecimientos mediáticos de alcance mundial y, por medio de ellos y gracias a su difusión por los medios de comunicación, al menos durante la celebración de dichos actos las ciudades anfitrionas acaparan la atención mundial. ${ }^{6}$

Además de fortalecer el nombre de la ciudad y su repercusión internacional, los eventos ańaden una componente intangible a la estructura física de la ciudad: una narrativa concreta y una forma de experimentar el espacio; en este contexto, la búsqueda y satisfacción de experiencias se ha configurado en un vector clave de la actual "economía simbólica" (Allen, 2002; Lash \& Urry, 1994), "economía del entretenimiento" (Bauman, 2007; Ritzer, 2006) o "economía de la experiencia” (Pine \& Gilmore, 1999).

Por todos estos motivos, se considera relevante la utilización de los eventos y/o festivales como indicadores estadísticos en la aproximación a la dimensión cultural de las ciudades. Debe aclararse que estos eventos no están midiendo la cultura, sino un tipo específico de manifestaciones ligadas a las estrategias culturales de la fase actual del capitalismo (Amin \& Thrift, 2007). Ellos constituyen, en todo caso, parámetros medibles en términos de acontecimientos celebrados y asistentes a los mismos, al tiempo que son servicios ofrecidos en formatos muy uniformes que se consumen mundialmente. Tal planteamiento, que recuerda el "empresarialismo de la gestión urbana” (Harvey, 1989) o la "mercantilización de la cultura”

$5 \longdiv { \text { Esta corriente crítica se detiene, además, en los problemas de seguridad y corrupción que pueden } }$ ocasionar estos eventos, como por ejemplo los ataques terroristas en los Juegos Olímpicos de Múnich (1972) y los escándalos de corrupción en los de Salt Lake City (2002). Junto a estos problemas, cabe mencionar la potencial infrautilización posevento de las instalaciones que, ante la falta de planificación, pueden quedar obsoletas (Baade \& Matheson, 2002).

6 Este fortalecimiento de la imagen de destino que sigue a un evento ha sido referido como el "efecto halo" (Hall, 1992), el "efecto escaparate" (Fredline \& Faulkner, 1998) y el "efecto que anima o feelgood” (Allen, O’Toole, McDonnell \& Harris, 2002). 
(Bauman, 2007), puede también ser objeto de críticas, en el sentido de que gran parte de las manifestaciones que se consideran pertenecen a las prácticas ligadas a la cultura occidental (música, cine, deportes, exposiciones, museos, etcétera), aspecto en cualquier caso controvertido dentro de la Ciencia Social (Nederveen Pieterse, 2003). A pesar de ello, y aceptando las limitaciones del análisis, los indicadores se consideran representativos, ya que las manifestaciones culturales occidentalizadas son predominantes en la escala global, sobre todo si la aproximación a ellas se realiza en términos cuantificables. Por otra parte, estos indicadores están ampliamente aceptados en algunos sistemas estadísticos, como en el de la Unesco (2009), que propone una serie de indicadores culturales globales en su Marco de Estadísticas, los mismos que sirven de referencia fundamental en la fase empírica de este trabajo.

\section{Metodología de la investigación}

A grandes rasgos, para llevar a cabo la evaluación de las ciudades en relación con sus funciones (ciudades multifuncionales vs. ciudades especializadas) se emplean dos índices sintéticos: uno de megaeventos (ISMeg), realizado expresamente para este artículo, y otro de carácter económico, tomado de los resultados ofrecidos por el equipo de investigación del Gawc (Global and World Cities Research Network), de la Universidad de Loughborought (Reino Unido). Lo denominaremos índice sintético del Gawc (Gawc, 2010).

La metodología utilizada en la realización del Ismeg se basa en el acopio de eventos y festivales de interés cultural sugeridos por la Unesco (2009) para la evaluación cultural de las ciudades. A diferencia de una gran parte de trabajos recientes, se caracteriza por ser una metodología abierta donde no existe una selección previa de ciudades, sino que el listado se ha ido construyendo sucesivamente a medida que se iban utilizando diferentes eventos e iban apareciendo nuevas ciudades. Las principales fases de su confección se resumen en:

a. Documentación y selección de información. Recopilación de 33 rankings urbanos para evaluar cada uno de los eventos culturales de que consta el Ismeg. Estos rankings son seleccionados por gozar del reconocimiento internacional del organismo elaborador. Las fechas oscilan de 2008 a 2014, según los casos, como modo de obtener resultados lo más actuales posibles (salvo en los Juegos Olímpicos y Exposiciones Universales, que se cuantifican desde el final de la Segunda Guerra Mundial). Los eventos, contabilizados por áreas metropolitanas, se agrupan en: megaeventos, conciertos de música, congresos internacionales, exposiciones de arte, festivales de cine y otros eventos deportivos. En la tabla 1 se sintetizan los indicadores, fuentes y unidades consideradas. 
TABLA I | Síntesis de los indicadores y fuentes utilizadas en la elaboración del IsMeg

\begin{tabular}{|c|c|c|c|}
\hline $\begin{array}{l}\text { MODALIDAD } \\
\text { DE EVENTO }\end{array}$ & INDICADOR & FUENTE & UNIDADES \\
\hline \multirow{4}{*}{ Megaeventos } & \multirow[t]{2}{*}{ Juegos Olímpicos } & \multirow[t]{2}{*}{$\begin{array}{l}\text { Página web oficial http://www. } \\
\text { olympic.org/ }\end{array}$} & $\begin{array}{l}\text { Sedes de Juegos Olímpico } \\
\text { verano e invierno tras Segunda } \\
\text { Guerra Mundial. }\end{array}$ \\
\hline & & & Ciudades candidatas desde 1992. \\
\hline & $\begin{array}{l}\text { Exposiciones } \\
\text { universales }\end{array}$ & $\begin{array}{l}\text { Bureau of International } \\
\text { Expositions (BIE) }\end{array}$ & $\begin{array}{l}\text { No de celebraciones tras Segun- } \\
\text { da Guerra Mundial. }\end{array}$ \\
\hline & $\begin{array}{l}\text { Campeonatos } \\
\text { mundiales de fútbol }\end{array}$ & $\begin{array}{l}\text { Página web oficial http://es.fifa. } \\
\text { com/worldcup/index.html }\end{array}$ & $\begin{array}{l}\text { No finales mundiales desde } \\
\text { Segunda Guerra Mundial. }\end{array}$ \\
\hline \multirow{3}{*}{$\begin{array}{l}\text { Conciertos } \\
\text { de música }^{(a)}\end{array}$} & $\begin{array}{l}\text { Actuaciones } \\
\text { de ópera }\end{array}$ & $\begin{array}{l}\text { Páginas web oficiales de cada } \\
\text { artista }\end{array}$ & $\begin{array}{l}\text { No de actuaciones de Renée } \\
\text { Fleming, Natalie Dessay, Anna } \\
\text { Netrebko, Juan Diego Flórez, } \\
\text { Plácido Domingo y Jonas Kaufa- } \\
\text { mann, desde } 2008 \text { a } 2013 \text {. }\end{array}$ \\
\hline & Conciertos de pop & $\begin{array}{l}\text { Páginas web oficiales de cada } \\
\text { artista }\end{array}$ & $\begin{array}{l}\text { No de actuaciones de Madonna, } \\
\text { Jeniffer López, Justin Bieber, } \\
\text { Rihanna, Lady Gaga, Britney } \\
\text { Spears, en sus respectivas giras } \\
\text { mundiales, desde } 2008 \text { a } 2012 .\end{array}$ \\
\hline & Conciertos de rock & $\begin{array}{l}\text { Páginas web oficiales de cada } \\
\text { grupo }\end{array}$ & $\begin{array}{l}\text { No de actuaciones de Eagles, } \\
\text { AcDC, Aerosmith, Rolling } \\
\text { Stones, Bruce Springsteen y } \\
\text { Metallica, en sus respectivas giras } \\
\text { mundiales, desde } 2008 \text { a } 2012 \text {. }\end{array}$ \\
\hline \multirow[b]{2}{*}{$\begin{array}{l}\text { Congresos } \\
\text { internacionales }\end{array}$} & \multirow[b]{2}{*}{$\begin{array}{l}\text { Congresos } \\
\text { internacionales }\end{array}$} & \multirow{2}{*}{$\begin{array}{l}\text { Asociación Internacional de } \\
\text { Congresos y Convenciones } \\
\text { (International Congress and } \\
\text { Convention Association [ICCA], } \\
2010 \text { ) }\end{array}$} & No asistentes. \\
\hline & & & No eventos internacionales. \\
\hline \multirow{2}{*}{$\begin{array}{l}\text { Exposiciones } \\
\text { de arte }\end{array}$} & \multirow{2}{*}{ Exposiciones de arte } & \multirow{2}{*}{ The Art Newspaper, 2010} & Asistencia media diaria. \\
\hline & & & No exposiciones. \\
\hline \multirow{3}{*}{$\begin{array}{l}\text { Festivales } \\
\text { de cine }\end{array}$} & \multirow{3}{*}{ Festivales de cine } & \multirow{3}{*}{$\begin{array}{l}\text { Database "Film Festival World", } \\
2012\end{array}$} & No de asistentes. \\
\hline & & & $\begin{array}{l}\text { No de medios de comunicación } \\
\text { acreditados. }\end{array}$ \\
\hline & & & No películas proyectadas. \\
\hline \multirow{4}{*}{$\begin{array}{l}\text { Otros eventos } \\
\text { deportivos }^{(b)}\end{array}$} & Fútbol americano & $\begin{array}{l}\text { Página web oficial http://www. } \\
\text { nfl.com/superbowl }\end{array}$ & No finales Super Bowl. \\
\hline & $\begin{array}{l}\text { Gran Premio de } \\
\text { Fórmula } 1\end{array}$ & $\begin{array}{l}\text { Página web oficial http://www. } \\
\text { formula1.com/ }\end{array}$ & No circuitos Campeonato FI. \\
\hline & $\begin{array}{l}\text { Campeonato Mun- } \\
\text { dial de Atletismo }\end{array}$ & $\begin{array}{l}\text { Página web oficial http://www. } \\
\text { iaaf.org/ }\end{array}$ & No de finales. \\
\hline & $\begin{array}{l}\text { Master series del } \\
\text { circuito ATP de tenis }\end{array}$ & $\begin{array}{l}\text { Página web oficial http://www. } \\
\text { atpworldtour.com }\end{array}$ & $\begin{array}{l}\text { No certámenes anuales (Inclui- } \\
\text { dos Grand Slams, Master 1000, } \\
\text { Master } 500 \text { y Master 250). }\end{array}$ \\
\hline
\end{tabular}

NOTAS (a) PARA LA SELECCIÓN DE CANTANTES EN CADA UNO DE LOS GÉNEROS MUSICALES CONSIDERADOS, SE UTILIZARON DIFERENTES REVISTAS ESPECIALIZADAS QUE VALORAN LA RELEVANCIA DE ESTAS FIGURAS; ENTRE ELLAS, LA REVISTA LIMELIGHT (2006) PARA EL GÉNERO DE OPERA; Y LAS REVISTAS FORBES (2OII) Y ROLLING STONE (2OI I), PARA LOS GÉNEROS POP-ROCK. (b) SE SELECCIONAN ESTOS DEPORTES POR CONSIDERAR QUE SON LOS QUE RECIBEN MAYOR AUDIENCIA TELEVISIVA (INITIATIVE FUTURES, SPORT + ENTERTAINMENT [IFSE], 2009)

FUENTE ELABORACIÓN PROPIA 
b. Proceso de normalización. Los indicadores que componen cada una de las modalidades se normalizan, con el fin de que sean homogéneos y comparables entre sí. Para ello, se utiliza el índice z, que normaliza los valores que adquieren los diferentes eventos en cada ciudad al restarles el valor medio y dividirlos por su desviación típica, lo que aminora los sesgos relacionados con distribuciones apuntadas (Peńa, 2001).

c. Asignación de ponderaciones discriminantes. Los eventos y festivales culturales utilizados han sido evaluados de diferente manera, a partir de ponderaciones discriminantes que valoran el grado de representatividad de cada uno de ellos. Todas las modalidades de eventos han sido ponderadas con un $15 \%$ cada una, salvo los megaeventos que, por su mayor representatividad e influencia internacional, han sido valorados con un $25 \%$.

d. Obtención del ISMeg. Los valores ponderados del índice z se ordenan jerárquicamente de mayor a menor, y se convierten en datos discretos (ordinales) para la obtención del ranking final. Se obtiene un índice sintético de 253 ciudades, aunque se acota en 150 (Anexo I) para poder llevar a cabo el análisis integrado con el IsGawc. ${ }^{7}$

Después de obtener el ISMeg, el objetivo consiste en cruzar este ranking con el índice sintético del Gawc (ISGawc) para evaluar la funcionalidad de los núcleos, valorando su carácter multifuncional o especializado, y el puesto que ocupan en los diferentes rankings. Al unificar los dos índices, se observa cómo algunas ciudades no tienen representación en un determinado ranking. En estos casos, se les asigna el valor 151; con este valor se incluyen aquellas ciudades que no están presentes en el índice original, o aquellas que, aun incluidas, están presentes pero por debajo de la posición 150; es decir, no figuran entre las 150 primeras, independientemente de si se posicionan o no en el índice original.

Para realizar este análisis cruzado de los dos índices jerárquicos disponibles, se ha empleado un análisis clúster. ${ }^{8}$ Su utilización se ajusta a los fines que se pretenden en esta investigación, ya que un análisis clúster clasifica una muestra de entidades (individuos o variables) en un número pequeño de grupos, de forma que las observaciones pertenecientes a un grupo son muy similares entre sí. Una dificultad a la hora de aplicar esta técnica es que el cálculo estadístico no determina automáticamente el número de grupos o clústeres, sino que el análisis requiere que se vaya experimentando con los resultados hasta que estos resulten relevantes desde el punto de vista de la interpretación de los datos. Se trata de una metodología muy difundida en

Este ranking de componente económico y gran reconocimiento internacional, evalúa los flujos de servicios avanzados a la producción en las ciudades del mundo, definidos como máximos exponentes de la globalización económica (Taylor, 1997). El índice clasifica las ciudades en Alfa, Beta y Gamma, en función del grado de conectividad en ese tipo de flujos. Debido a que sus datos son de naturaleza relacional (índice de conectividad), y los del ismeg son de naturaleza estática (número de eventos), se decidió convertir ambos listados en valores discretos para poder llevar a cabo un análisis integrado de ambos índices, evitando la variabilidad de los datos.

8 Mediante el software informático spss Statistics 19.0, utilizando el algoritmo de K-medias. 
análisis estadísticos para determinar el comportamiento grupal de diferentes variables (Córdoba \& Gago, 2010; Gago \& Córdoba, 2013).

Esta aproximación cruzada de los índices tendrá un especial interés cuando se trate de señalar aquellas ciudades que destacan por albergar eventos de índole cultural. Muchas de ellas coincidirán con las ciudades establecidas en el IsGawC, pero otras muchas no lo harán. Nuestro interés se centra en destacar ambas casuísticas: (i) ciudades coincidentes en ambos índices: ciudades generalmente "globales" que destacan por su carácter multifuncional y que se ajustan, por tanto, a las definiciones de ciudad global formuladas por diferentes autores (Friedman, Sassen, Taylor, Castells); interesará evaluar el grado de variación y superioridad que la dimensión cultural, a través de los eventos, confiere a muchas ciudades del sistema mundo; (ii) ciudades no coincidentes: se trata de ciudades no registradas en los índices sobre el sistema urbano mundial de corte más económico y que aparecen en el Ismeg, evidenciando una absoluta especialización funcional en esta componente; por consiguiente y vista su importancia, este hecho lleva a insistir en la necesidad de incorporar estas variables en los estudios urbanos.

Esta primera parte del análisis pretende identificar conjuntos de ciudades (nombrados con números arábigos) donde, además de aquellas tradicionalmente cartografiadas, aparezcan otras que también pueden considerarse de orden global, porque albergan estrategias representativas en la sociedad de ocio-consumo contemporánea. En este sentido, el resultado pretende validar la hipótesis de partida de este trabajo, que sostiene que la jerarquía urbana mundial está siendo modelada por funciones culturales además de las de tipo económico, configurándose así un archipiélago más complejo.

Identificadas las ciudades económicas y/o culturales, la segunda parte de la investigación se detiene en la especialización funcional de las ciudades exclusivamente desde el punto de vista cultural, atendiendo a los eventos y festivales deportivos y/o culturales desarrollados por las mismas. Utilizando los valores ponderados obtenidos de cada ciudad para cada una de las modalidades de eventos consideradas, se evalúa el grado de especialización de las ciudades a partir del coeficiente de localización de Sargent-Florence: $\mathrm{CL}=(\mathrm{Eij} / \mathrm{Ei}) /(\mathrm{Ej} / \mathrm{Et})$, donde:

- Eij: Valor ponderado que representa un evento determinado en una ciudad (\%).

- Ej: Valor total que alcanza una determinada ciudad en todos los eventos.

- Ei: Valor total que representa un evento determinado en todas las ciudades consideradas.

- Et: Valor total que alcanzan todos los eventos analizados en todas las ciudades consideradas (1).

En aquellos casos donde el citado coeficiente muestre valores superiores a $1(>1)$, se considera que la ciudad en cuestión está especializada en la celebración de un determinado evento, el cual desempeña un papel fundamental no solo en la identidad de la ciudad, sino sobre todo en su proyección internacional, desde una perspectiva cultural contextualizada en la "economía cultural". 
Estos coeficientes de especialización son sometidos a un nuevo análisis clúster para agrupar las ciudades por grandes conjuntos de especialización funcional. Los resultados se exponen nuevamente por conjuntos de ciudades (identificados con letras mayúsculas) donde, además de explicar el significado y relevancia de estos eventos para las ciudades anfitrionas, se ofrece una síntesis cartográfica de la especialización funcional de las ciudades según la modalidad de eventos que acogen.

\section{Análisis comparativo de los índices ISMeg e IsGaWC}

En términos absolutos, las veinte primeras ciudades del índice sintético de megaeventos (ISMeg) se localizan predominantemente en Europa Occidental y América del Norte, seguidas de otras regiones mundiales, como Oceanía, América Latina y Asia (Anexo I). Las primeras evidencias se observan al analizar conjuntamente el Top 20 de ambos índices (IsGawc e Ismeg) (figura 1).

En primer lugar, se evidencia la falta de correlación que existe entre ambos rankings, pues tan solo 8 de las 20 primeras ciudades del IsMeg aparecen también en el ISGawc. Esta afirmación se corrobora realizando el coeficiente de correlación de Spearman, que sitúa la correlación en 0,15 para las 20 primeras ciudades de ambos índices. Esta baja correspondencia pone de manifiesto, a nuestro juicio, cómo la celebración de eventos y festivales responde a iniciativas de naturaleza política con una elevada voluntad decisoria que, aunque en un principio no demandan economías de aglomeración, sí las pretenden generar a futuro.

En segundo lugar, Nueva York, Londres, París y Tokio lideran ambos índices, aunque con leves variaciones de rango en torno a una o dos posiciones. Constituyen las grandes ciudades globales por excelencia, con las que existe un acuerdo generalizado en la literatura académica por presentarlas en las posiciones más elevadas de casi todos los rankings urbanos (Sassen, 1991; Friedmann, 1995; Gawc, 2010).

Además, aparecen grandes ascensos del ISMeg respecto al ISGawC, superiores incluso a 30 posiciones. Es el caso Berlín (+52), Barcelona (+33), Montreal (+42), Roma (+42), Berna (+140), Río de Janeiro (+73) y Estocolmo (+134). Salvo Barcelona, se trata de ciudades de segundo nivel desde el punto de vista económico, pero que experimentan una gran variación positiva desde la perspectiva cultural.

Otras ciudades son muy relevantes en el apartado económico, pero están mejor posicionadas en el Ismeg. Su buen rango en ambos índices contribuye a que las variaciones no sean tan evidentes como en el punto anterior: Los Ángeles (+14).

Por otro lado, se hallan otras ciudades donde los eventos también desempeñan un rol muy importante, aunque supeditado al indicador económico (la diferencia ISMeg/ISGawC es negativa). Estas ciudades constituyen los centros económicos de sus respectivos países y grandes regiones mundiales a las que pertenecen; ellas son Hong Kong (-38), Singapur (-28), Shanghái (-18), Beijing (-23) y Mumbai (-135) en Asia; Chicago (-34) y Toronto (-10) en América del Norte; Dubái (-85) en Oriente Próximo; Milán (-25), Moscú (-8), Frankfurt (-32) en Europa; y la Ciudad de México (-7) en América Latina. 
FIGURA I | Variaciones de rangos en el Top 20 del Ismeg respecto al ISGawC ISGaWC

ISMeg

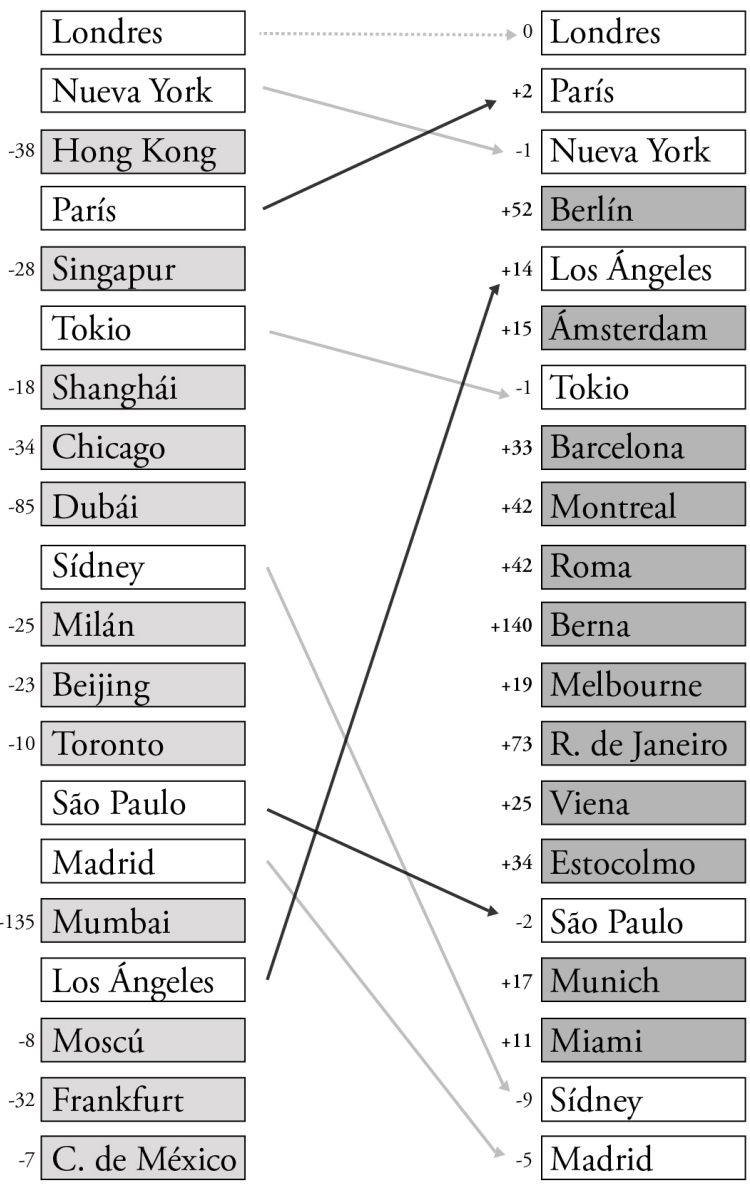

NOTA SE ASOCIA EL GRIS OSCURO A LAS VARIACIONES POSITIVAS A FAVOR DE LOS EVENTOS, BIEN EN FLECHAS (POR ESTAR EN EL TOP 20 DEL ISGAWC), BIEN EN RECUADROS (POR ESTAR POR DEBAJO DE LAS VEINTE PRIMERAS DEL ISGAWC). POR EL CONTRARIO, EL GRIS ClaRo SE ASOCIA A LAS VARIACIONES POSITIVAS A FAVOR DE LA VARIABLE ECONÓMICA

FUENTE ELABORACIÓN PROPIA

Aunque existen variaciones en las primeras 20 posiciones, las mayores diferencias empiezan a generalizarse a medida que se desciende en el ranking. De hecho, en su mayor parte los autores no se ponen de acuerdo respecto de la jerarquía urbana mundial, exceptuando las ciudades que coronan la misma (Beaverstock, Smith \& Taylor, 1999). Las ciudades de la cúspide están muy definidas; el problema aparece cuando la atención se aleja de esa cúspide, pues en función de los criterios que se tenga en consideración, aparecerán unas ciudades u otras. Para evaluar estas diferencias, se propone una representación gráfica en un eje cartesiano (figura 2), donde se 
analiza el ISmeg (eje x) y el ISGawC (eje y) a través de los rangos absolutos en que se posicionan las ciudades en ambos índices.

En ese gráfico se señalan, además, los principales grupos de ciudades obtenidos, una vez aplicado el análisis clúster a ambos rankings. Aunque en un principio se obtuvieron diez conglomerados urbanos, para su correcto entendimiento se ha considerado relevante una reagrupación de los mismos en función de las características de los conjuntos.

FIGURA 2 Distribución de las ciudades en función de la posición en los índices ISMeg e ISGawC

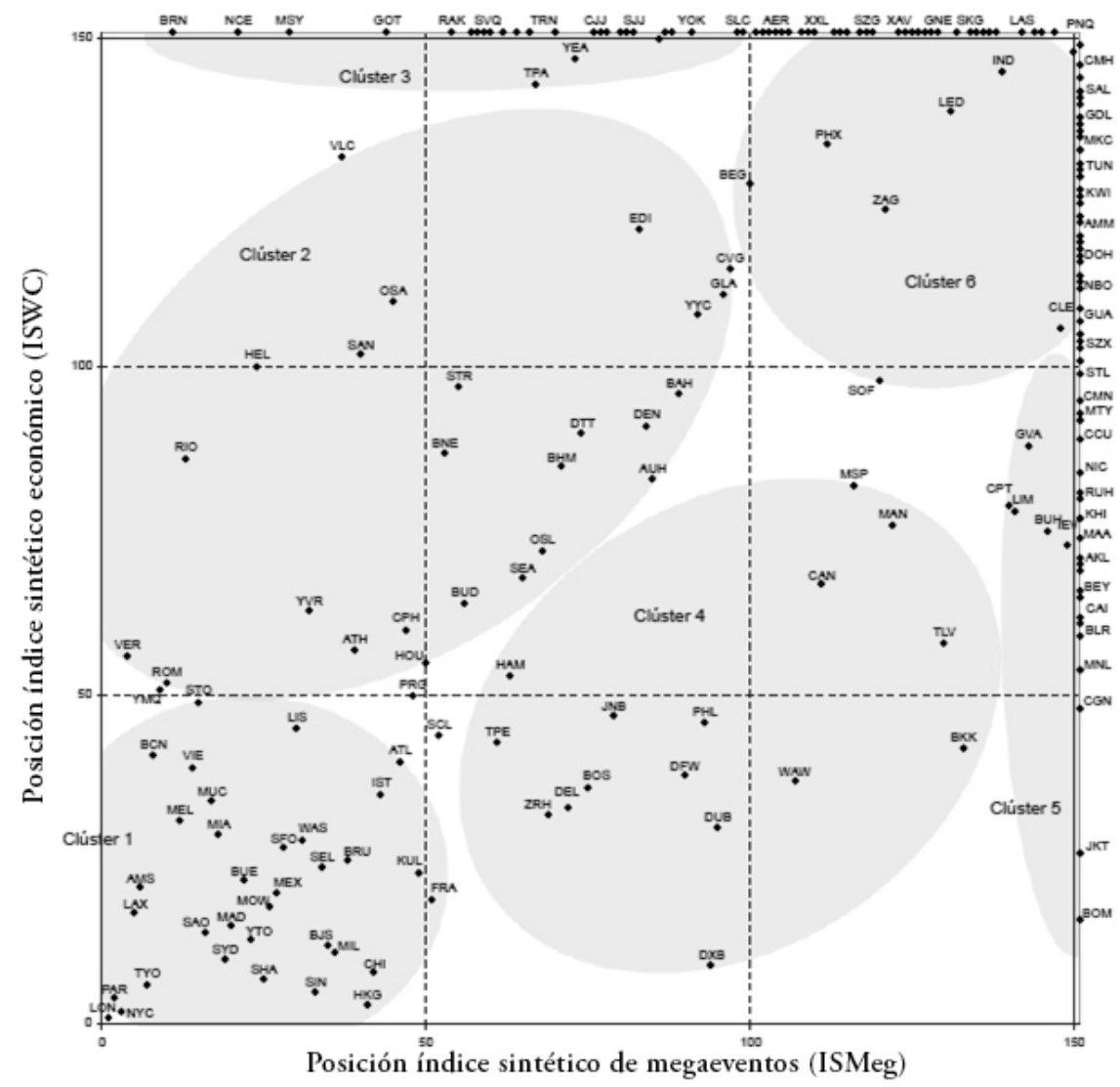

NOTA ABREVIATURAS SE CORRESPONDEN CON LOS CÓDIGOS DE IDENTIFICACIÓN DE LA IATA (ASOCIACIÓN INTERNACIONAL DEL TRANSPORTE AÉREO (WWW.IATA.ORG)

FUENTE ELABORACIÓN PROPIA

Clúster 1. Este grupo está integrado por 35 ciudades localizadas en Europa, América del Norte y Asia, principalmente. Las incluidas en este grupo se posicionan en los rangos más elevados de sus respectivos índices (tabla 2); en ambos aparecen en el Top 50 urbano (rango medio Ismeg 21,4; rango medio IsGawC 24,88). Se consideran 
ciudades globales, que no solo lideran el gobierno de sus respectivos países, sino que trascienden fronteras para actuar en la órbita internacional, sometidas a intensos procesos de competencia. Debido a esta notoriedad, gran parte de la literatura científica les atribuye, además, una competitividad muy elevada, y mantenida en forma constante con el objeto de no alejarse demasiado de los puestos principales.

TABLA 2 Variación de rangos del índice ISMeg respecto al ISGawC del grupo 1

\begin{tabular}{|c|c|c|c|c|c|c|}
\hline \multirow{2}{*}{$\begin{array}{l}\text { RANGO } \\
\text { ISMeg }\end{array}$} & \multirow{2}{*}{$\begin{array}{l}\text { RANGO } \\
\text { ISGAWC }\end{array}$} & \multirow[b]{2}{*}{ CIUDAD } & \multirow[b]{2}{*}{ PAÍS } & \multirow[b]{2}{*}{ ABREVIATURA } & \multicolumn{2}{|c|}{ VAR. RANGO ISGaWC-ISMeg } \\
\hline & & & & & $\begin{array}{c}\mathrm{N}^{\mathrm{o}} \\
\text { POSICIONES }\end{array}$ & $\%$ VAR. \\
\hline 1 & 1 & Londres & Reino Unido & LON & 0 & 0 \\
\hline 2 & 4 & París & Francia & PAR & 2 & 50 \\
\hline 3 & 2 & Nueva York & EE.UU. & NYC & -1 & -50 \\
\hline 5 & 17 & Los Ángeles & EE.UU. & LAX & 12 & 70,58 \\
\hline 6 & 21 & Ámsterdam & Países Bajos & AMS & 15 & 71,42 \\
\hline 7 & 6 & Tokio & Japón & TYO & -1 & $-16,66$ \\
\hline 8 & 41 & Barcelona & España & $\mathrm{BCN}$ & 33 & 80,48 \\
\hline 12 & 31 & Melbourne & Australia & MEL & 19 & 61,29 \\
\hline 14 & 39 & Viena & Austria & VIE & 25 & 64,10 \\
\hline 15 & 49 & Estocolmo & Suecia & STO & 34 & 69,38 \\
\hline 16 & 14 & São Paulo & Brasil & $\mathrm{SAO}$ & -2 & $-14,28$ \\
\hline 17 & 34 & Múnich & Alemania & MUC & 17 & 50 \\
\hline 18 & 29 & Miami & EE.UU. & MIA & 11 & 37,93 \\
\hline 19 & 10 & Sídney & Australia & SYD & -9 & -90 \\
\hline 20 & 15 & Madrid & España & MAD & -5 & $-33,33$ \\
\hline 22 & 22 & Buenos Aires & Argentina & BUE & 0 & 0 \\
\hline 23 & 13 & Toronto & Canadá & YTO & -10 & $-76,92$ \\
\hline 25 & 7 & Shanghái & China & SHA & -18 & $-257,14$ \\
\hline 26 & 18 & Moscú & Rusia & MOW & -8 & $-44,44$ \\
\hline 27 & 20 & C. de México & México & MEX & -7 & -35 \\
\hline 28 & 27 & San Francisco & EE.UU. & SFO & -1 & $-3,70$ \\
\hline 30 & 45 & Lisboa & Portugal & LIS & 15 & 33,33 \\
\hline 31 & 28 & Washington & EE.UU. & WAS & -3 & $-10,71$ \\
\hline 33 & 5 & Singapur & Singapur & SIN & -28 & -560 \\
\hline 34 & 24 & Seúl & Corea & SEL & -10 & $-41,66$ \\
\hline 35 & 12 & Beijing & China & BJS & -23 & $-191,67$ \\
\hline 36 & 11 & Milán & Italia & MIL & -25 & $-227,27$ \\
\hline 38 & 25 & Bruselas & Bélgica & BRU & -13 & -52 \\
\hline 41 & 3 & Hong Kong & Hong Kong & $\mathrm{HKG}$ & -38 & $-1266,7$ \\
\hline 42 & 8 & Chicago & EE.UU. & $\mathrm{CHI}$ & -34 & -425 \\
\hline 43 & 35 & Estambul & Turquía & IST & -8 & $-22,85$ \\
\hline 46 & 40 & Atlanta & EE.UU. & ATL & -6 & -15 \\
\hline 48 & 50 & Praga & Rep. Checa & PRG & 2 & 4 \\
\hline 49 & 23 & Kuala Lumpur & Malasia & KUL & -26 & $-113,04$ \\
\hline 51 & 19 & Frankfurt & Alemania & FRA & -32 & $-168,42$ \\
\hline
\end{tabular}

NOTA LAS CIUDADES SE AGRUPAN NO SOLO POR SU NIVEL DE VARIACIÓN, SINO ANTE TODO POR SU POSICIONAMIENTO EN EL ÍNDICE ISMEG; EN GRIS SE REPRESENTAN AQUELLAS CIUDADES COINCIDENTES CON CIUDADES DE PRIMER NIVEL DEL ISGAWC

FUENTE ELABORACIÓN PROPIA 
Al tratarse de ciudades globales, la diferencia de posiciones es bastante reducida, puesto que se intuye una notoriedad en la globalización de carácter multifuncional. Estas ciudades coinciden con aquellas que lideran los rankings realizados sobre sistemas urbanos mundiales como el ISGawC, y otros también de naturaleza económica (Alderson \& Beckfield, 2004; Global Economic Power Index, 2012). Generalmente existen pocas variaciones de unos índices a otros, dada la notoriedad de las ciudades globales (Gago \& Pisonero, 2013); es lo que Duarte y Ultramari (2007) denominan como "inflexiones urbanas". Esta diferencia es inferior a 10 posiciones para los siguientes casos: Londres (0 posiciones), Nueva York (-1), París (+2), Tokio (-1), San Francisco (-1), São Paulo (-2), Washington (-3), Madrid (-5), Atlanta (-6), México (-7), Estambul (-8), Moscú (-8), Sídney (-9) y Praga (+2).

Dentro de este gran grupo se podrían diferenciar, a su vez, dos subgrupos: el primero estaría integrado por aquellas ciudades que alcanzan un mayor rango en ISMeg respecto al ISGawC. Se trata de importantes nodos urbanos con un gran potencial de producción y consumo cultural; en concreto, de megaeventos como estrategias de diferenciación, dinamización y citymarketing. Como se señalaba anteriormente, son en estas ciudades premium donde se sospechan importantes efectos acumulativos, al aprovecharse economías de escala, beneficiarse de la adecuada accesibilidad y del capital humano que en ellas reside, así como por disponer de un gran número de habitantes, especialmente aquellos considerados creativos, que son los que consumen cultura de manera más notable. Las diferencias más importantes se dan en Barcelona (+33 posiciones), Viena $(+25)$, Melbourne $(+19)$, Múnich $(+17)$, Ámsterdam (+15), Lisboa (+15), Los Ángeles (+12) o Miami (+11).

En el lado opuesto, existen otras ciudades muy importantes en la esfera global, pero en las que la componente económica las sitúa en puestos jerárquicos superiores al indicador de los megaeventos. Todas ellas coinciden con ciudades de primer nivel del ISGawC, que, tal como las define este grupo de investigación, albergan un gran número de sedes de grandes empresas de servicios avanzados. Además de algunos de los nodos económicos más importantes de Europa (Frankfurt, Milán y Bruselas) y América del Norte (Chicago y Toronto), el análisis de las diferencias entre ambos índices evidencia la emergencia manufacturera de algunos países de la región asiática y el desplazamiento del peso de gravedad en las actividades productivas hacia oriente. Destacan los ascensos respecto al Ismeg de Hong Kong (-38), Singapur (-28), Kuala Lumpur (-26), Beijing (-23) y Seúl (-10). Esto no significa que estos nodos sean únicamente polos económicos -todo lo contrario-, sino que las dimensiones analizadas las sitúan en un rango alto en todas ellas.

Clúster 2. A partir de este grupo, empieza a darse un mayor grado de especialización entre las ciudades integrantes. Incluye nodos de cierta trascendencia mundial, ya que en los dos indicadores funcionales analizados están relativamente bien posicionadas (intervalo 9-97 en el ISMeg; 51-147 en el ISGawC), aunque se evidencia una significativa especialización del indicador de los megaeventos (tabla 3). Europa y América del Norte siguen siendo las regiones predominantes; Asia y Latinoamérica aparecen de manera anecdótica, con Osaka y Río de Janeiro, respectivamente.

En este grupo urbano, de menor diversificación geográfica que el clúster 1, el nivel de especialización cultural es tan elevado que las ciudades integrantes constituyen 
algunos de los exponentes culturales más significativos de sus respectivas regiones mundiales, a los que cabe suponer cierta proyección en la escala global, especialmente para el caso de Berlín, Montreal, Roma, Río de Janeiro, Helsinki, Vancouver, Valencia, San Diego y Osaka (todas ellas en el Top 50 del Ismeg y con una diferencia entre índices superior a 30 posiciones), e incluso para los ejemplos de Brisbane, Stuttgart, Tampa, Edmonton, Edimburgo y Oporto (por debajo del Top 50, pero también con grandes variaciones entre los dos índices). Por tanto, puede señalarse que dentro del grupo 2 se identifican algunos de los nodos urbanos donde la estrategia funcional en la celebración de eventos culturales confiere un estatus privilegiado en el mapa global de orden cultural, gracias a los patrones de producción y consumo cultural instaurados en la sociedad contemporánea.

TABLA 3 | Variación de rangos del índice ISMeg respecto al ISGaWC del grupo 2

\begin{tabular}{|c|c|c|c|c|c|c|}
\hline \multirow{2}{*}{$\begin{array}{l}\text { RANGO } \\
\text { ISMeg. }\end{array}$} & \multirow{2}{*}{$\begin{array}{l}\text { RANGO } \\
\text { ISGaWC }\end{array}$} & \multirow[b]{2}{*}{ CIUDAD } & \multirow[b]{2}{*}{ PAÍS } & \multirow[b]{2}{*}{ ABREVIATURA } & \multicolumn{2}{|c|}{ VAR. RANGO ISGAWC-ISMeg } \\
\hline & & & & & $\begin{array}{c}\mathrm{N}^{\mathbf{0}} \\
\text { POSICIONES }\end{array}$ & $\%$ VAR. \\
\hline 4 & 56 & Berlín & Alemania & BER & 52 & 92,85 \\
\hline 9 & 51 & Montreal & Canadá & YMQ & 42 & 82,35 \\
\hline 10 & 52 & Roma & Italia & ROM & 42 & 80,76 \\
\hline 13 & 86 & Río de Janeiro & Brasil & RIO & 73 & 84,88 \\
\hline 24 & 100 & Helsinki & Finlandia & HEL & 76 & 76 \\
\hline 32 & 63 & Vancouver & Canadá & YVR & 31 & 49,20 \\
\hline 37 & 132 & Valencia & España & VLC & 95 & 71,96 \\
\hline 39 & 57 & Atenas & Grecia & ATH & 18 & 31,57 \\
\hline 40 & 102 & San Diego & EE.UU. & SAN & 62 & 60,78 \\
\hline 45 & 110 & Osaka & Japón & OSA & 65 & 59,09 \\
\hline 47 & 60 & Copenhague & Dinamarca & $\mathrm{CPH}$ & 13 & 21,66 \\
\hline 50 & 55 & Houston & EE.UU. & HOU & 5 & 9,090 \\
\hline 53 & 87 & Brisbane & Australia & BNE & 34 & 39,08 \\
\hline 55 & 97 & Stuttgart & Alemania & STR & 42 & 43,29 \\
\hline 56 & 64 & Budapest & Hungría & BUD & 8 & 12,5 \\
\hline 65 & 68 & Seattle & EE.UU. & SEA & 3 & 4,41 \\
\hline 67 & 143 & Tampa & EE.UU. & TPA & 76 & 53,14 \\
\hline 68 & 72 & Oslo & Noruega & OSL & 4 & 5,55 \\
\hline 71 & 85 & Birmingham & EE.UU. & внм & 14 & 16,47 \\
\hline 73 & 147 & Edmonton & Canadá & YEA & 74 & 50,34 \\
\hline 74 & 90 & Detroit & EE.UU. & DTT & 16 & 17,77 \\
\hline 83 & 121 & Edimburgo & Reino Unido & EDI & 38 & 31,40 \\
\hline 84 & 91 & Denver & EE.UU. & DEN & 7 & 7,69 \\
\hline 86 & 150 & Oporto & Portugal & OPO & 64 & 42,66 \\
\hline 89 & 96 & Manama & Bahréin & BAH & 7 & 7,29 \\
\hline 92 & 108 & Calgary & Canadá & YYC & 16 & 14,81 \\
\hline 96 & 111 & Glasgow & Reino Unido & GLA & 15 & 13,51 \\
\hline 97 & 115 & Cincinnati & EE.UU. & CVG & 18 & 15,65 \\
\hline
\end{tabular}

NOTA LAS CIUDADES SE AGRUPAN NO SOLO POR SU NIVEL DE VARIACIÓN, SINO ANTE TODO POR SU POSICIONAMIENTO EN EL ÍNDICE ISMEG; EN GRIS SE RESALTAN AQUELLAS CIUDADES CON UNA VARIACIÓN DE RANGOS ISGAWC/ISMEG SUPERIOR A $5 O$ POSICIONES

FUENTE ELABORACIÓN PROPIA 
Clúster 3. Un tercer grupo de ciudades se caracteriza por su elevado grado de especialización en la celebración de eventos deportivos y/o culturales como estrategia de dinamización cultural. A diferencia del grupo antecedente, el grado de especialización es más elevado, porque las variaciones son muy considerables en términos absolutos (tabla 4). Su notoriedad cultural es muy significativa, no solo por el rango en el que se desenvuelven (intervalo 11-99), sino porque no aparecen en el ranking económico (o se hallan muy relegadas en él). Suele tratarse de nodos y subnodos de carácter regional, no presentes en los rankings urbanos más tradicionales de carácter económico, que se caracterizan por desempeñar un rol muy específico en las dinámicas globales, aunque su rango puede hacer sospechar un alcance superior en alguno de ellos. Las diferencias entre posiciones resultan muy elevadas, evidenciando también un elevado grado de excelencia cultural. Los casos más ilustrativos aparecen donde la diferencia entre ambos índices es superior a 50 posiciones y, además, gozan de una posición privilegiada en el índice sintético de megaeventos: Berna (\#11), Niza (\#21), Nueva Orleans (\#29), Gotemburgo (\#44), Marrakech (\#54), Sevilla (\#57), Daegu (\#58), Nagoya (\#59), Génova (\#60), San Sebastián (\#62) o Venecia (\#64), entre otras.

TABLA 4 Variación de rangos del índice ISMeg respecto al ISGawC del grupo 3

\begin{tabular}{|c|c|c|c|c|c|c|}
\hline \multirow[b]{2}{*}{$\begin{array}{l}\text { RANGO } \\
\text { ISMeg. }\end{array}$} & \multirow[b]{2}{*}{$\begin{array}{l}\text { RANGO } \\
\text { ISGaWC }\end{array}$} & \multirow[b]{2}{*}{ CIUDAD } & \multirow[b]{2}{*}{ PAÍS } & \multirow[b]{2}{*}{ ABREVIATURA } & \multicolumn{2}{|c|}{ VAR. RANGO ISGaWC-ISMeg } \\
\hline & & & & & $\begin{array}{c}\mathrm{N}^{\mathbf{o}} \\
\text { POSICIONES }\end{array}$ & \% VAR. \\
\hline 11 & 151 & Berna & Suiza & BRN & 140 & 92,71 \\
\hline 21 & 151 & Niza & Francia & NCE & 130 & 86,09 \\
\hline 29 & 151 & Nueva Orleans & EE.UU. & MSY & 122 & 80,79 \\
\hline 44 & 151 & Gotemburgo & Suecia & GOT & 107 & 70,86 \\
\hline 54 & 151 & Marrakech & Marruecos & RAK & 97 & 64,23 \\
\hline 57 & 151 & Sevilla & España & SVQ & 94 & 62,25 \\
\hline 58 & 151 & Daegu & Corea del Sur & TAE & 93 & 61,58 \\
\hline 59 & 151 & Nagoya & Japón & NGO & 92 & 60,92 \\
\hline 60 & 151 & Génova & Italia & GOA & 91 & 60,26 \\
\hline 62 & 151 & San Sebastián & España & EAS & 89 & 58,94 \\
\hline 64 & 151 & Venecia & Italia & VCE & 87 & 57,61 \\
\hline 66 & 151 & Austin & EE.UU. & AUS & 85 & 56,29 \\
\hline 70 & 151 & Turín & Italia & TRN & 81 & 53,64 \\
\hline 76 & 151 & Daejeon & Corea del Sur & $\mathrm{CJJ}$ & 75 & 49,66 \\
\hline 77 & 151 & Plovdiv & Bulgaria & PDV & 74 & 49,00 \\
\hline 78 & 151 & Basilea & Suiza & BSL & 73 & 48,34 \\
\hline 80 & 151 & Gwangju & Corea del Sur & KWJ & 71 & 47,01 \\
\hline 81 & 151 & Busan & Corea del Sur & PUS & 70 & 46,35 \\
\hline 82 & 151 & Sarajevo & Bosnia y Her. & SJJ & 69 & 45,69 \\
\hline 87 & 151 & Liege & Bélgica & LGG & 64 & 42,38 \\
\hline 88 & 151 & Clermont-Ferr. & Francia & CFE & 63 & 41,72 \\
\hline 91 & 151 & Yokohama & Japón & YOK & 60 & 39,73 \\
\hline 98 & 151 & Salt Lake City & EE.UU. & SLC & 53 & 35,09 \\
\hline 99 & 151 & Bilbao & España & BIO & 52 & 34,43 \\
\hline FUENTE & $\begin{array}{l}\text { LAS CIUD } \\
\text { POSICION } \\
\text { CON UNA } \\
\text { SE PRESEI } \\
\text { ELABORAC }\end{array}$ & $\begin{array}{l}\text { ADES SE AGRUPAN } \\
\text { AMIENTO EN EL } \\
\text { VARIACIÓN DE RA } \\
\text { JTAN AQUELLAS } \\
\text { IÓN PROPIA }\end{array}$ & $\begin{array}{l}\text { NO SOLO POR } \\
\text { NDICE ISMEG; } \\
\text { TGOS ISGAWC/I } \\
\text { PRESENTES E }\end{array}$ & $\begin{array}{l}\text { U NIVEL DE VAR } \\
\text { N GRIS CLARO } \\
\text { MEG SUPERIOR } \\
\text { J EL TOPI } 5 O \text { DE }\end{array}$ & $\begin{array}{l}\text { ACIÓN, SINO A } \\
\text { E RESALTAN AQ } \\
50 \text { POSICIONES } \\
\text { ISMEG }\end{array}$ & $\begin{array}{l}\text { TODO POR SU } \\
\text { AS CIUDADES } \\
\text { GRIS OSCURO }\end{array}$ \\
\hline
\end{tabular}


La importancia de estas ciudades en esta investigación radica en su invisibilidad en otros rankings, bien por haber sido elaborados con metodologías "cerradas", basadas en una preselección de ciudades, bien por emplear indicadores que inciden en la economía. Estas ciudades con mayor grado de variación son las que precisamente evidencian un superior nivel de especialización funcional y, por consiguiente, argumentan la necesidad de evaluar la globalización desde una perspectiva multidimensional.

Por limitaciones de espacio, no se entra a analizar el resto de grupos urbanos ( $4 \mathrm{y}$ 5 , donde el indicador económico es superior al indicador de los eventos culturales; y 6 , donde ambos indicadores se posicionan en rangos atrasados por debajo del umbral \#100). A nuestro juicio, los tres primeros grupos son los que mejor demuestran las hipótesis planteadas al comienzo de la investigación: por un lado, el carácter multifuncional de las ciudades globales (grupo 1); y por otro, el carácter más especializado en componentes culturales (megaeventos) de ciudades secundarias que van más allá de la perspectiva económica (grupos 2 y 3 ).

Desde el punto de vista geográfico, se pone de manifiesto cómo la distribución de los eventos adquiere especial interés en las regiones desarrolladas, predominantemente en el mundo occidental, siguiendo el patrón de distribución de los servicios avanzados. Cabe suponer que las tendencias culturales de carácter anglosajón y/u occidental, según la metodología empleada, parecen extenderse como, al menos, algunas de las prácticas culturales del globo. Sin embargo, el desplazamiento del poder económico que se está produciendo hacia Asia-Pacífico no se traduce en un desarrollo de las estrategias culturales asociadas a los eventos, al menos no con la intensidad que los servicios avanzados están representando en los denominados países emergentes. Muchas de sus ciudades, especialmente de China e India, parecen priorizar los criterios económicos sobre los culturales en su posicionamiento dentro de la jerarquía urbana mundial.

\section{Especialización funcional de las ciudades atendiendo al tipo de evento de acogida}

El análisis comparado de ambos índices ha permitido no solo diferenciar grandes conjuntos de ciudades en función de su comportamiento en el funcionamiento general de ambos indicadores (económico y eventos culturales), sino sobre todo poner de manifiesto la necesidad de considerar este tipo de servicios culturales en el estudio del sistema urbano mundial. Debido a la notoriedad que representan estas estrategias culturales, la investigación se detiene, a continuación, en el conocimiento particular de la especialización funcional de las ciudades por grandes conjuntos, a partir del tipo de evento que acogen para proyectarse internacionalmente. Se aprovecha, asimismo, de explicar el significado y relevancia de estos eventos para las ciudades anfitrionas.

Conviene matizar que, aunque los eventos considerados en este trabajo son en su mayoría periódicos, aparecen otros muy puntuales de orden mundial cuya excepcionalidad los hace únicos (megaeventos). La celebración de estos últimos no implica que la ciudad anfitriona esté especializada en este tipo de eventos; sin 
embargo, la exposición de los resultados se refiere en tal sentido, a fin de facilitar la compresión de los mismos. Se considera que, pese a su carácter puntual, estos eventos tienen gran importancia, pues proyectan a la ciudad internacionalmente, su recuerdo perdura en el imaginario colectivo al ser reiteradamente recordados en los medios de comunicación, y dejan una impronta muy importante en la fisionomía de la ciudad.

Una vez obtenidos los coeficientes de especialización para cada una de las ciudades mediante el coeficiente de localización de Sargent-Florence, y su posterior agrupamiento estadístico utilizando de nuevo la técnica del clúster (véase metodología), se obtienen siete conglomerados de ciudades que representan las ya mencionadas event strategies de cada una de las ciudades que integran el ranking ISMeg. Los resultados se exponen a continuación.

Grupo A. Este grupo de carácter "multievento" se subdivide en dos subgrupos. El primero (subgrupo A1; figura 3a) está integrado por 33 ciudades y constituye la agrupación más relevante, pues en ella figuran aquellas ciudades posicionadas en el Top 50 (o muy próximo a este umbral) del ismeg. En su mayor parte, coinciden con el grupo 1 del apartado anterior. La competitividad de estas ciudades es tan elevada que se ven obligadas a albergar varios tipos de eventos de trascendencia internacional, con coeficientes de especialización superiores a 1 en, al menos, tres modalidades de eventos. La notoriedad de estas ciudades abarca un amplio espectro de actividades consideradas de orden mundial, no solo de orden cultural, que es precisamente lo que confiere a estos nodos su estatus global. Entre otras, cabe destacar Londres, París, Nueva York o Tokio, consideradas las ciudades globales por antonomasia en gran parte de los rankings urbanos. Junto a ellas, otras como Berlín, Roma, Río de Janeiro o Estocolmo, adquieren gran calado internacional en temas culturales vinculados con los megaeventos. Precisamente, el carácter "multievento" de estas ciudades es uno de los factores prioritarios que permiten escalar a las mismas respecto a otros rankings urbanos más tradicionales, de naturaleza económica, y, por consiguiente, proyectarlas en la esfera global de índole cultural.

El segundo subgrupo (subgrupo A2; figura 3b) consta de 10 ciudades que albergan múltiples eventos pero, a diferencia del subgrupo anterior (A1), las ciudades gozan de un menor alcance global (por estar posicionadas por debajo del rango \#50).

El resto de conglomerados incluye ciudades de menor alcance que, a diferencia del grupo A, se caracterizan por un mayor nivel de especialización en un determinado evento, que es el que define en mayor medida la estrategia de proyección cultural adoptada por la ciudad. Aunque algunas ciudades pueden tener representatividad en dos o incluso tres acontecimientos, solo uno de ellos contribuye a definir en mayor medida la identidad "eventful" de la ciudad. Los grupos más importantes se examinan a continuación. 
FIGURA 3 | Caracterización funcional de las ciudades del grupo A, con base en la (a y b) modalidad de eventos que acogen (se representan los valores medios del coef. de localización para cada grupo de ciudades)
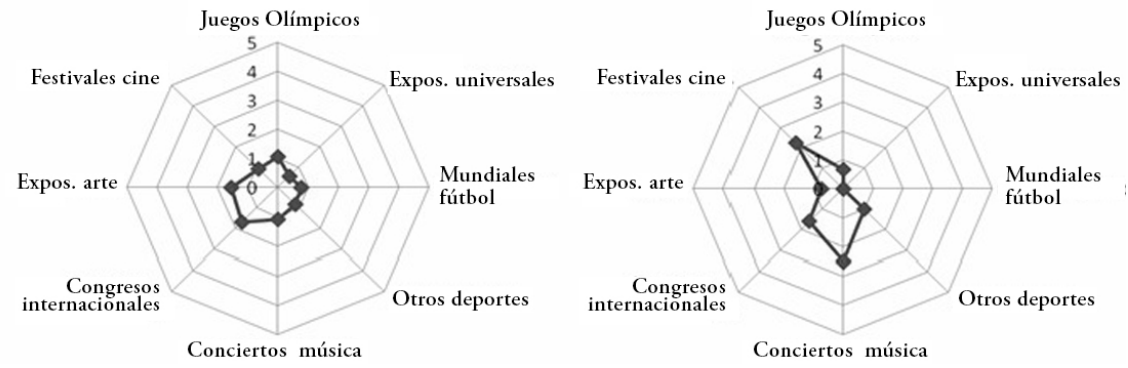

\begin{tabular}{|l|l|}
\hline Grupo A1: & $\begin{array}{l}\text { Londres, París, Nueva York, Berlín, Los Ángeles, Ámsterdam, Tokio, Barcelona, Montreal, } \\
\text { Roma, Melbourne, Río de Janeiro, Viena, Estocolmo, Múnich, Sidney, Toronto, Moscú, } \\
\text { San Francisco, Lisboa, Vancouver, Seúl, Beijing, Milán, Valencia, Chicago, Estambul, } \\
\text { Atlanta, Copenhague, Praga, Houston, Frankfurt, Hamburgo. }\end{array}$ \\
\hline Grupo A2: & $\begin{array}{l}\text { Dallas, Dubái, Belgrado, Varsovia, Zagreb, Manchester, San Petesburgo, Leipzig, Ciudad } \\
\text { del Cabo, Ginebra. }\end{array}$ \\
\hline
\end{tabular}

NOTA CUANDO EL COEFICIENTE DE LOCALIZACIÓN ES SUPERIOR A I SIGNIFICA QUE LA CIUDAD (EN NUESTRO CASO, GRUPO DE CIUDAdes) ESTÁ ESPECIALIZADA EN LA CELEBRACiÓN DE UN DETERMINADO EVENTO

FUENTE ELABORACIÓN PROPIA

Grupo B. Acoge aquellas ciudades que han sido anfitrionas de un evento de grandes dimensiones, conocido comúnmente como "megaevento". Como se ha seńalado con anterioridad, los más singulares son los Juegos Olímpicos, las Exposiciones Universales y las finales de los mundiales de fútbol, pues se trata de los eventos culturales públicos por excelencia más visibles y espectaculares de la sociedad moderna (Davis, 2012; Roche, 2000). En este grupo B se diferencian tres subgrupos, según sea el evento particular que define la estrategia funcional de la ciudad anfitriona (figura 4; grupos B1, B2 y B3). En todos los casos, estos megaeventos han facilitado la proyección de las ciudades integrantes en el mapa cultural desde la perspectiva de los eventos (Getz, 2008; Maenning \& Zimbalist, 2012).

Grupo C. Este conglomerado está definido por la gran especialización en la celebración de conciertos musicales de gran trascendencia internacional (figura 4; grupo C). Algunos autores reconocen la música como una estrategia de branding (en inglés, music branding), muy vinculada al concepto del marketing de eventos (Krüger, 2013), especialmente si estos eventos son protagonizados por grandes estrellas mundiales capaces de liderar ingentes cantidades de seguidores y/o fans. Este grado de idolatría permite instaurar los denominados lovemarks (Roberts, 2004), con los que se fundamentan las emociones y experiencias, especialmente en directo, ante la consolidación de la denominada "economía de la experiencia" (Pine \& Gilmore, 1999). Aunque son varios los géneros musicales incluidos en el estudio, generalmente es el género de la ópera el que concede esta especialización musical (Zúrich, Boston, Filadelfia, Salzburgo, Marsella, Bucarest), aunque también otras ciudades adquieren gran repercusión gracias a la celebración de conciertos pop-rock de estrellas internacionales (Dublín, Las Vegas, entre otras). 
FIgURA 4 | Caracterización funcional de las ciudades especializadas en la celebración de un determinado evento (valores medios del coeficiente de localización para cada grupo)

\begin{tabular}{|c|c|}
\hline $\begin{array}{c}\text { GRUPO BI. MEGAEVENTOS (ESPECIALIZACIÓN } \\
\text { EN JUEGOS OLÍMPICOS) }\end{array}$ & $\begin{array}{r}\text { GRUPO B2. MEGAEVENTOS (ESPECIALIZACIÓN } \\
\text { EN EXPOSICIONES UNIVERSALES) }\end{array}$ \\
\hline $\begin{array}{l}\text { Festivales cine } \\
\text { Expos. arte }\end{array}$ & $\begin{array}{l}\text { os. universales } \\
\text { Mundiales } \\
\text { fútbol }\end{array}$ \\
\hline $\begin{array}{l}\text { Oslo, Turín, Sarajevo, Denver, Calgary, Salt } \\
\text { Lake City, Pyonyang, Sapporo, Sochi, Cortina } \\
\text { d'Ampezzo, Lillehammer, Insbruck, Grenoble, } \\
\text { Albertville, Lake Placid, Nagano, Squaw Valley, } \\
\text { St. Moritz. }\end{array}$ & $\begin{array}{l}\text { Shanghái, Bruselas, Osaka, Brisbane, Sevilla, } \\
\text { Nagoya, Génova, Seattle, Daejeon, Yokohama, } \\
\text { San Antonio, Zaragoza, Hannover, Knoxville y } \\
\text { Spokane. }\end{array}$ \\
\hline
\end{tabular}

\begin{tabular}{|l|l|}
$\begin{array}{c}\text { GRUPO B3. MEGAEVENTOS (ESPECIALIZACIÓN } \\
\text { EN MUNDIALES DE FÚTBOL) }\end{array}$ & $\begin{array}{c}\text { GRUPO C. ESPECIALIZACIÓN EN CONCIERTOS } \\
\text { DE MÚSICA }\end{array}$ \\
\hline & Expos. universales
\end{tabular}



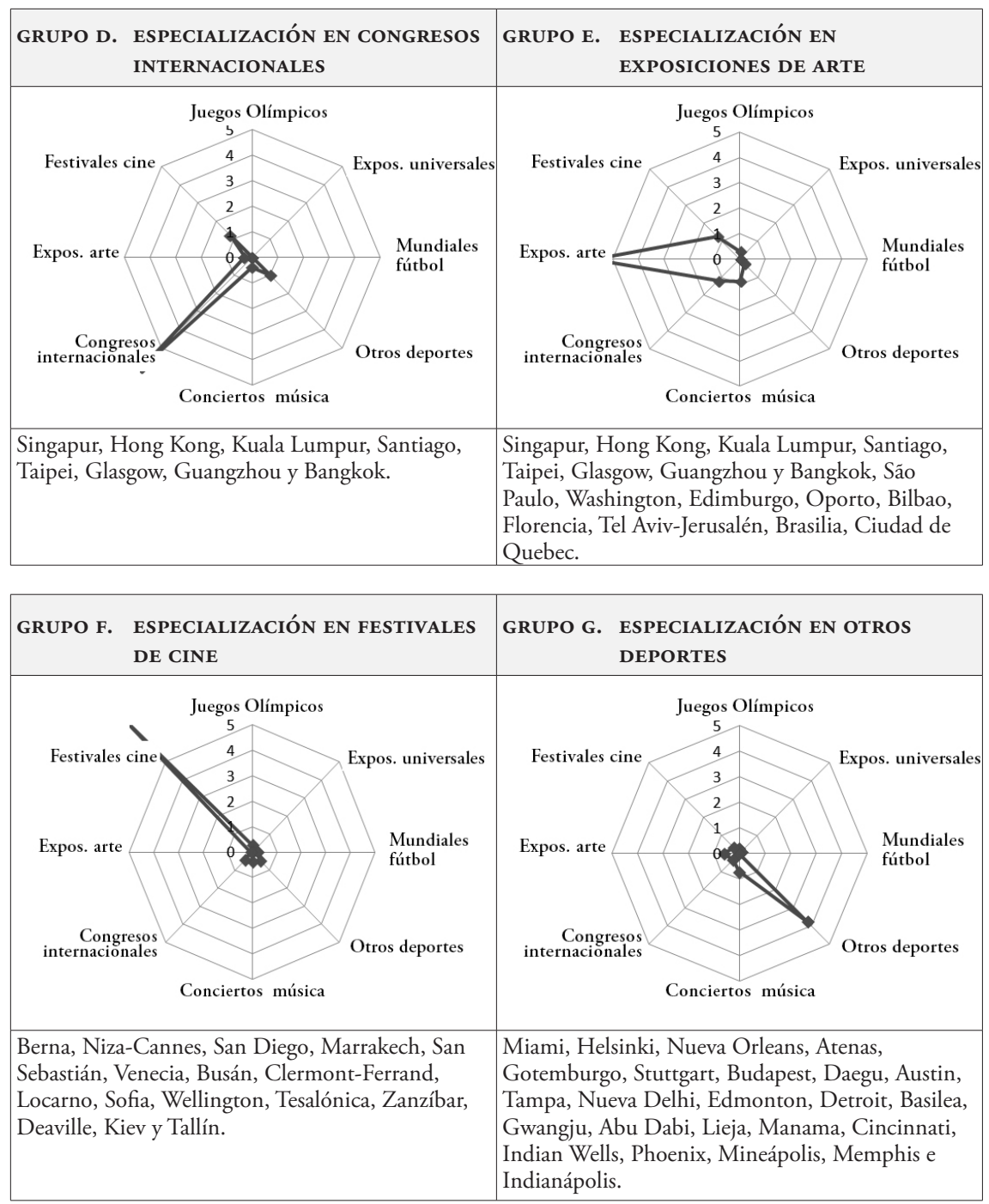

NOTA CUANDO EL COEFICIENTE DE LOCALIZACión ES SUPERIOR A I SigNifica QUe LA CIUDAD (EN NUESTRO CASO, GRUPO DE CIUDADES) ESTÁ ESPECIALIZADA EN LA CELEBRACIÓN DE UN DETERMINADO EVENTO

FUENTE ELABORACIÓN PROPIA 
Grupo D. Otro grupo de ciudades adquiere especial interés en la celebración de congresos internacionales (figura 4, grupo D), uno de los segmentos de mercado más dinámicos del turismo contemporáneo: el turismo profesional (Davidson $\&$ Cope, 2003). Estos eventos no solo proporcionan importantes beneficios económicos, sino que, además, sus palacios de congresos, en muchos casos emblemáticos, así como la movilidad global que subyace entre sus asistentes, confieren prestigio y proyección internacional, lo que convierte el turismo profesional en una pieza fundamental dentro de las actuales estrategias de citymarketing (Swarbrooke \& Horner, 2001). Se pone de manifiesto cómo las capitales asiáticas, nodos de primer nivel en los servicios avanzados, se especializan en estos eventos de reuniones ante su constante crecimiento en el escenario global, en consonancia con el desplazamiento del poder económico mundial hacia el este.

Grupo E. Este grupo de ciudades desempeña un rol muy significativo en el ámbito del arte, al acoger algunas de las exhibiciones (pintura y escultura) de mayor asistencia diaria a nivel mundial (figura 4, grupo E). Además de los consolidados recintos museísticos internacionales (milla de los museos de Nueva York o Londres, y paseo del arte de Madrid, entre otros), aparecen otras ciudades muy especializadas en este tipo de actos: São Paulo, Washington, Edimburgo, Oporto y Bilbao, entre otras. Aunque no todos los museos tienen la misma representatividad ni ejercen el mismo impacto sobre la ciudad en que se asientan, lo cierto es que aquellos más importantes están experimentando una evolución funcional y de forma, al ser utilizados como mecanismos de promoción de la competitividad urbana y de reforzamiento de la identidad local (Rausell, 2007; Millán, 2009).

Grupo F. Este grupo utiliza los festivales de cine como importantes herramientas de citymarketing para promocionar las ciudades anfitrionas en el mapa mundial de orden cultural (figura 4, grupo F). Aunque en la actualidad existen más de 400 festivales de cine reconocidos de talla internacional que se celebran cada año (Kenneth, 2002), solo unos pocos han sabido posicionar a sus ciudades anfitrionas como las "Mecas del cine" en el mapa global. Estas son Niza-Cannes (\#21) y Venecia (\#64), principalmente, cuyos festivales son considerados de clase Premium por la Federación Internacional de Productores Cinematográficos (Federation of Film Producers Associations [FIAPF], 2012). Otras ciudades se alzan en esta competitividad urbana buscando su identidad mediante la especialización de sus festivales: Berna (\#11) y su festival de cine de cortometrajes; San Diego (\#40) y su festival de cine independiente y San Sebastián (\#62), también catalogado de Tipo A por la FIAPF.

Grupo G. El último grupo aglutina aquellas ciudades especializadas en la celebración de un evento deportivo, que no sean los Juegos Olímpicos ni el mundial de fútbol (catalogados estos como megaeventos). En concreto, se refiere a los cuatro deportes de mayor audiencia televisiva mundial (IFSE, 2009): el campeonato mundial de futbol americano, el campeonato mundial de atletismo, el campeonato de automovilismo Fórmula 1 y los diferentes certámenes que componen el circuito mundial ATP de tenis (figura 4, grupo G). Al igual que otros eventos deportivos, estos cuatro campeonatos tienen un gran significado para las ciudades, no solo a 
nivel económico, social y urbano, sino, especialmente, de diferenciación e imagen urbana (Bradley \& Hall, 2006; Davis, 2012).

En síntesis, se evidencia cómo los eventos deportivos y/o culturales permiten proyectar y singularizar a las ciudades anfitrionas en el sistema urbano mundial bajo una perspectiva cultural contextualizada en la sociedad del ocio y consumo de masas. Estos efectos no solo se sienten en las denominadas ciudades multifuncionales (grupo A), sino también en aquellas otras más especializadas (resto de grupos), que tratan de definir su identidad "eventful" para competir y alzarse en la propuesta de jerarquía urbana de componente cultural cimentada en la celebración de eventos culturales.

\section{Conclusiones}

El objetivo de ejecutar un estudio más abierto y global, que no relegara ninguna de las áreas que pudieran influir en el funcionamiento real de la globalización, ha llevado a defender una conceptualización de la misma que también afecte a esferas que son intangibles en el marco del capitalismo cultural cognitivo (Scott, 2008). En este sentido, el discurso teórico de este trabajo ha sostenido que los componentes funcionales de matiz cultural, representados a través de la celebración de eventos y festivales culturales, constituyen nuevas bases de competencia urbana y son totalmente necesarios para un completo entendimiento de la globalización; por consiguiente, es inevitable su introducción en los estudios urbanos si se quiere determinar ajustadamente la organización del archipiélago metropolitano mundial.

Aunque se reconoce la limitación metodológica de cuantificar eventos celebrados durante un periodo de tiempo concreto (2008-2014) y se acepta que los resultados se podrían ver alterados en función del tipo de variables consideradas, se considera que la hipótesis inicial se ha validado empíricamente, al demostrar que la notoriedad de una ciudad deriva no solo de su carácter estrictamente económico, sino que actúan otras funciones paralelas que dan sentido a la perspectiva multidimensional. Asimismo, los resultados han puesto de manifiesto la necesidad de detenerse no solo en las ciudades globales, sino también aquellas otras secundarias caracterizadas por un mayor grado de especialización funcional. Estas últimas, a pesar de sus alcances de menor envergadura que las ciudades globales, contribuyen a plasmar un sistema urbano mundial más complejo y variado que lo esperado inicialmente. Así, unas y otras (globales y secundarias) ponen de manifiesto cómo su proyección en el mapa global se adapta a la escala y posibilidades de cada una. Por un lado, las ciudades de mayor influencia internacional compiten por ser anfitrionas de eventos de gran envergadura y repercusión global; precisamente los megaeventos son los más eficaces para el posicionamiento de las ciudades no solo por la magnitud que alcanza la regeneración urbana, sino, especialmente, por su repercusión en los medios de comunicación. Por otro lado, las ciudades más pequeñas apuestan por eventos más modestos de carácter regional, aunque en ocasiones con un gran calado global, como los festivales de cine de Venecia, Cannes o San Diego, la ópera de Salzburgo, los conciertos en directo de Las Vegas, o las exposiciones de arte en reconocidas pinacotecas como las de Bilbao o Florencia. 
Este tipo de actividades culturales, tal como se ha demostrado con los índices de correlación entre los dos índices analizados (ISMeg e ISGawC), responden de manera importante a iniciativas cargadas de una elevada voluntad política, en las cuales priman los criterios de rentabilidad económica o visibilización del núcleo urbano. En el actual contexto neoliberal, los políticos o dirigentes urbanos trazan estrategias para que las ciudades que representan sean anfitrionas de eventos importantes, como camino para que las ciudades continúen posicionándose en el mapa global y también para garantizar una imagen urbana de prestigio que permita singularizar a las mismas respecto al resto de ciudades.

Asimismo, la distribución geográfica de estos eventos ha puesto en evidencia cómo la competencia creciente de las ciudades por albergar este tipo de actividades predomina en aquellas urbes localizadas en el mundo desarrollado y/u occidental. Esto no quiere decir que su actuación se focalice exclusivamente aquí, pero sí en mayor grado. En este sentido, cabe destacar cómo el basculamiento del poder económico hacia el Lejano Oriente no está implicando un desarrollo de las estrategias culturales en estas ciudades, al menos no con la intensidad de los servicios avanzados (salvo en las grandes capitales asiáticas). Sin embargo, conviene reconocer que la actuación de estos eventos empieza a extrapolarse, levemente, a las áreas emergentes y en vías de desarrollo, que esperan de ellos un efecto catalizador de su economía y de su proyección simbólica en la esfera global.

Llegados hasta aquí, cabe preguntarse si un campeonato del mundo, un festival de cine internacional o cualquier otro gran evento, conceden la categoría global a una ciudad. De lo que no cabe duda es que contribuyen de manera manifiesta tanto a la proyección de las ciudades anfitrionas hacia el exterior en la búsqueda de todo tipo de movilidades (inmigrantes, turistas, trabajadores cualificados, inversiones, ideas), como a su dinámica interna, que responde, más que nunca, a demandas de ocio, turismo y consumo de masas. Por ello, aunque en cierto modo la distribución de estos eventos refuerza la notoriedad de las ciudades del norte, se considera necesario seguir invirtiendo en ellos como estrategias de dinamización, citymarketing y branding urbano, para abrir nuevas oportunidades a otras ciudades del archipiélago urbano mundial, bien se trate de ciudades secundarias (desde el punto de vista escalar), bien de ciudades del sur (desde el punto de vista geográfico).

En conclusión, se estima que estos eventos se han convertido en un factor importante del proceso de globalización cultural de las ciudades y, por ende, se creen válidos en la aproximación empírica a la dimensión cultural pues, a nuestro juicio, son representativos de las industrias culturales que ayudan a las ciudades, entre otras cosas, a abastecerse del capital simbólico, experiencial, de ocio y, en definitiva, cultural, que son vitales en el éxito competitivo de la "nueva economía" o "economía cultural". 


\section{Referencias bibliográficas}

Alderson, A. \& Beckfield, J. (2004). Power and position in the world city system. American Journal of Sociology, 109(4), 811-851. http://dx.doi.org/10.1086/378930

Allen, J. (2002). Symbolic economies: The 'culturalization' of economic knowledge. En P. Gay \& M. Pryke, (Eds.), Culture, representation and identities: Cultural economy: Cultural analysis and commercial life (pp. 39-59). Londres: Sage.

Allen, J., O’Toole, W., McDonnell, I. \& Harris, R. (2002). Festival and special event management. Londres: Wiley.

Amin, A. \& Thrift, N. (2007). Cultural-economy and cities. Progress in Human Geography, 31(2), 143-161. doi: 10.1177/0309132507075361

Art Newspaper, The. (2011, April). Exhibition \& Museum attendance figures 2010. [En línea]. The Art Newspaper, n. ${ }^{\circ} 22$ [Museums], 23-30. http://www.museologie.uqam.ca/ Page/Document/art_newspaper_2011-04.pdf

Baade, R. \& Matheson, V. (2002). Bidding for the olympics: Fool's gold. En C. Barros, M. Ibrahimo, M. \& S. Szymanski (Eds.), Transatlantic sport: The comparative economics of North American and European sports (pp. 127-151). Chetelham, uk: Edward Elgar Publishing.

Bauman, Z. (2007). Vida de consumo. Buenos Aires: Fondo de Cultura Económica.

Beaverstock, J., Smith, R. \& Taylor, P. (1999). A roster of world cities. Cities, 16(6), 445-458. http://dx.doi.org/10.1016/S0264-2751(99)00042-6

Bou, E. (2009). Exposiciones universales: descubrimientos, utopías y marcas. Cultura y Ciudad, (14), 6.

Bradley, A. \& Hall, T. (2006). The festival phenomenon: Festivals, events and the promotion of small urban areas. En D. Bell \& M. Jayne, Small cities: Urban experience beyond the metropolis (pp. 77-90). Londres: Routledge.

Chan, A. \& Ross, R. (2002). From North-South to South-South: The true face of global competition. Foreign Affairs, 81(5), 8-13.

Córdoba, J. (Dir.), Babinger, F., Córdoba, M., Pisonero, R. D., De Marcos, J., Gago, C., Santander, F. \& Serrano, M. (2010). Atlas Interactivo del mundo, Parte III. Globalización y sostenibilidad, CD-Rom Interactivo, Madrid: Editorial Complutense.

Córdoba, J. \& Gago, C. (2010). Latin American cities and globalization: Change and permanency in the context of development expectations. Urban Studies, 47(9), 20032021. doi: $10.1177 / 0042098010372680$

Currid, E. (2006). New York as a global creative hub: A competitive analysis of four theories on world cities. Economic Development Quarterly, 20(4), 330-350. doi: $10.1177 / 0891242406292708$

Davidson, R. \& Cope, B. (2003). Business travel: Conferences, incentive travel, exhibitions, corporate hospitality and corporate travel. Nueva York: Pearson Education.

Davis, J. A. (2012). The Olympic Games effect: How sports marketing builds brands. Nueva York: Wiley.

Duarte, F. \& Ultramari, C. (2007). Inflexiones urbanas y ciudades globales: evidencias y jerarquías. [En línea]. Biblio $3 w$ [Revista Bibliográfica de Geografía y Ciencias Sociales], 12(743). http://www.ub.edu/geocrit/b3w-743.htm 
Essex, S. \& Chalkley, B. (2003). Urban transformation from hosting the Olympic Games: University lecture on the Olympics. [En línea]. Barcelona: Centre d'Estudis Olímpics (UAB), International Chair in Olympism (IOC-UAB). http://olympicstudies.uab.es/ lectures/web/pdf/essex.pdf

Evans, G. (2009). Creative cities, creative spaces and urban policy. Urban Studies, 46(5-6), 1003-1040. doi: 10.1177/0042098009103853

Federation of Film Producers Associations [Federación Internacional de Asociaciones de Productores Cinematográficos] (fIAPF). (2012). 'A' List Festivals. FilmFestivalLife. [En línea]. www.blog.filmfestivallife.com/09/13/a-list-festivals/

FilmFestivalWorld (2012). El mundo de los festivales de cine. [En línea, uRL cerrada en la actualidad]. Directorio estadístico de festivales de cine. http://www.filmfestivalworld. $\mathrm{com} /$

Florida, R. (2002). The rise of the creative class: and how it's transforming work, leiruse, community, and everyday life. Nueva York: Basic Books.

Florida, R. (2005). Cities and the creative class. Nueva York: Routledge.

Fredline, E. \& Faulkner, B. (1998). Resident reactions to a major tourist event: The Gold Coast Indy car race. Festival Management and Event Tourism, 5(4), 185-205. doi: $10.3727 / 106527098791784475$

Friedmann, J. (1995). Where we stand: A decade of world city research. En P. Knox \& P. Taylor (Eds.), World Cities in a World-System (pp. 21-47). Cambridge, Ru: Cambridge University Press. http://catdir.loc.gov/catdir/samples/cam041/94032444.pdf

Gaffney, C. (2010). Mega-events and socio-spatial dynamics in Rio de Janeiro, 1919-2016. Journal of Latin American Geography, 9(1), 7-29. doi: 10.1353/lag.0.0068

Gago, C. \& Córdoba, J. (2013). Sistemas urbanos en América Latina: roles y advenimientos de nuevos centros desde la perspectiva del transporte aéreo. Anales de Geografía de la Universidad Complutense, 33(2), 9-39. doi: 10.5209/rev_AGUC.2013.v33.n2.43000

Gago, C. \& Pisonero, R. D. (2013). Los rankings de ciudades globales: el papel de los servicios culturales. En J. Gutiérrez, A. Nieto, F. Jariz, E. Ruiz \& F. Anton (Eds.), Los servicios: dinámicas, infraestructuras y cohesión territorial (pp. 163-180). Cáceres, España: Asociación de Geógrafos Españoles.

García Canclini, N. (1995). Consumidores y ciudadanos. Conflictos multiculturales de la globalización. México, DF.: Grijalbo.

Getz, D. (2008). Event tourism: Definition and research. Tourism Management, 29(3), 403428. http://dx.doi.org/10.1016/j.tourman.2007.07.017

Global and World Cities Researh Group (Gawc). (2010). The World According to gawc 2010. [En línea]. Loughborough University, uK. http://www.lboro.ac.uk/gawc/ world2010t.html

Global Economic Power Index. (2012). The global cities that offer the most opportunity; America's most powerful global cities; Which cities have the most global clout; The 25 most economically powerful cities in the world. Artículos por R. Florida, en CITYLAB. [En línea]. http://www.citylab.com/topics/global-economic-power-index/

Groote, P. (2005). A multidisciplinary analysis of world fairs (= expos) and their effects. Tourism Review, 60(1), 12-19. http://dx.doi.org/10.1108/eb058448

Hall, C. (1992). Hallmark tourist events: Impacts, management and planning. Nueva York: Wiley. 
Harvey, D. (1989). The condition of postmodernity: An enquiry into the origins of cultural change. Camabridge, MA/Oxford, uk: Blackwell Pub. Versión digitalizada en http://bit. ly/2bnihmA

Herrero, L., Sanz, J., Devesa, M., Begate, A. \& Del Barrio, M. (2006). The economic impact of cultural events. A case-study of Salamanca 2002, European capital of culture. European Urban and Regional Studies, 13(1), 41-57. doi: 10.1177/0969776406058946

Initiative Futures, Sport + Entertainment (IFSE). (2009). The most watched TV sporting events of 2009. [En línea]. www.influencia.net/data/.../viewertrack-2010.pdf

International Congress and Convention Association [Asociación Internacional de Congresos y Convenciones] (ICCA). (2010). Statistic Report 2010. International Association Meetings Market. [En línea]. http://iccaworld.com/dcps/doc.cfm?docid=1246

Kenneth, T. (2002). Sundance to Sarajevo: Film festivals and the world they made. Berkeley, Ca: University of California Press.

Kim, H., Gursoy, D. \& Lee, S. (2006). The impact of the 2002 World Cup on South Korea: Comparisons of pre-and post-games. Tourism Management, 27(1), 86-96. http:// dx.doi.org/10.1016/j.tourman.2004.07.010

Krüger, S. (2013). Branding the city. Music tourism and the European capital of culture. En S. Krüger \& R. Trandafoui (Eds.), The globalization of musics in transit: Music migration and tourism (pp. 135-159). Londres: Routledge.

Lash, S. \& Urry, J. (1994). Economies of Signs and Space. Londres: Sage.

Lipovetsky, G. (2003). La era del vacio: Ensayos sobre el individualismo contemporáneo. Barcelona: Anagrama.

Lipovetsky, G. (2006). Los tiempos hipermodernos. Barcelona: Anagrama.

Lipovetsky, G. \& Serroy, J. (2015). La estatización del mundo. Vivir en la época del capitalismo artístico. Barcelona: Anagrama.

Maening, W. \& Zimbalist, A. (Eds.). (2012). International handbook on the economics of mega sporting events. Londres: Edward Elgar.

Mansvelt, J. (2008). Geographies of consumption: Citizenship, space and practice. Progress in Human Geography, 32(1), 105-117. doi: 10.1177/0309132507080623

Marcuse, P. \& Van Kempen, R. (2000). Globalizing cities. A new spatial order. Nueva York: Wiley.

Méndez, R. \& Sánchez, S. (2011). Spanish cities in the knowledge economy. Theoretical debates and empirical evidence. European Urban and Regional Studies, 18(2), 136-155. doi: $10.1177 / 0969776410381039$

Millán, R. (2009). Sistema global del arte: museos de arte contemporáneo, bienales y ferias como mecanismos de posicionamiento urbano en los circuitos globales. EURE, 35(106), 155-169. http://dx.doi.org/10.4067/S0250-71612009000300008

Nedeveen Pieterse, J. (2003). Globalization as hybridization. En R. Robertson \& K. White (Eds.), Globalization: Critical concepts in sociology (pp. 265-291). Londres: Taylor \& Francis.

Peña, D. (2001). Fundamentos de Estadistica. Madrid: Alianza.

Pine, B. \& Gilmore, J. (1999). The experience economy: Work is theatre \& every business a stage. Boston, MA: Harvard Business School Press. 
Power, D. \& Nielsen, T. (2010). Priority Sector Report: Creative and cultural industries. [En línea]. Europe Innova, European Cluster Observatory, European Commision. http:// www.diva-portal.org/smash/get/diva2:359744/FULLTEXT01.pdf

Precedo, A., Orosa, J. \& Míguez, A. (2010). De la planificación estratégica al marketing urbano: hacia la ciudad inmaterial. EURE, 36(108), 5-28. http://dx.doi.org/10.4067/ S0250-71612010000200001

Rausell, P. (2007). Museo y excelencia en las ciudades. En Museos y Ciudades. Nuevos escenarios para el desarrollo. XV Congreso Nacional de Amigos de los Museos, Valencia, 2-4 de marzo de 2007 (pp. 24-38). https://www.researchgate.net/publication/265411054_ Museos_y_excelencia_en_las_ciudades

Richards, G. (2007). The festivalization of society or the socialization of festivals? The case of Catalunya. En G. Richards (Ed.), Cultural tourism: Global and local perspectives (pp. 257-279). Binghampton: Haworth Hospitality Press.

Ritzer, G. (2006). La globalización de la nada. Madrid: Popular.

Roberts, K. (2004). Lovemarks: The future beyond brands. Nueva York: Powerhouse Books.

Roche, M. (2002). Megaevents and modernity: Olympics and expos in the growth of global culture. Londres: Routledge.

Romeiro, P. \& Méndez, R. (2008). Las ciudades del conocimiento: revisión crítica y posibilidades de aplicación a las ciudades intermedias. Scripta Nova, 12(270), 50. http://www.ub.edu/geocrit/sn/sn-270/sn-270-50.htm

Sánchez, S. (2013). Servicios y desarrollo urbano: una aproximación a las características del empleo en la "economía creativa" en la ciudad de Madrid. En J. Gutiérrez, A. Nieto, F. Jariz, E. Ruiz \& F. Anton (Eds.), Los servicios: dinámicas, infraestructuras y cohesión territorial (pp. 277-294). Cáceres, España: Asociación de Geógrafos Españoles.

Sassen, S. (1991). The Global City: New York, London, Tokyo. Princeton: Princeton University Press.

Scott, A .J. (2008). Social economy of the metropolis: Cognitive-cultural capitalism and the global resurgence of cities. Oxford: Oxford University Press.

Scott, A. (2009). Human capital resources and requirements across the metropolitan hierarchy of the USA. Journal of Economic Geography, 9(2), 207-226. doi: 10.1093/jeg/lbn051

Scott, A. (2010). Space-time variations of human capital assets across U.s. metropolitan areas, 1980 to 2000. Economic Geography, 86(3), 233-250.

Shatking, G. (2007). Global cities of the South: Emerging perspective on growth and inequality. Cities, 24(1), 1-15. http://dx.doi.org/10.1016/j.cities.2006.10.002

Storper, M. \& Scott, A. (2009). Rethinking human capital, creativity and urban growth. Journal of Economic Geography, 9, 147-167. doi: 10.1093/jeg/lbn052

Swarbrooke, J. \& Horner, S. (2001). Business travel and tourism. Londres: Routledge.

Taylor, P. (1997). Hierarchical tendencies amongst world cities: A global research proposal. Cities, 14, 323-332. http://dx.doi.org/10.1016/S0264-2751(97)00023-1

Tressera, J. (2004). La tematización de las ciudades: el uso de la cultura en las estrategias de desarrollo local y la promoción del turismo urbano. Turismo y Sociedad, 3, 71-85. http://revistas.uexternado.edu.co/index.php/tursoc/article/view/2226 
Unesco [Organización de las Naciones Unidas para la Educación, la Ciencia y la Cultura]. (2009). Marco de Estadísticas culturales de la Unesco 2009. [En línea]. Montreal, Quebec, Canadá: Instituto de Estadística de la Unesco. http://unesdoc.unesco.org/ images/0019/001910/191063s.pdf

Urry, J. (1990). The tourist gaze, leisure travel in contemporary societies. Londres: Sage.

Waitt, G. (2003). Social impacts of the Sydney Olympics. Annals of Tourism Research, 30(1), 194-215. http://dx.doi.org/10.1016/S0160-7383(02)00050-6

\section{Agradecimientos}

El autor agradece la ayuda recibida por parte de los profesores Juan Córdoba, Cándida Gago y Simón Sánchez Moral de la Universidad Complutense de Madrid, así como de los evaluadores externos que han enriquecido el contenido de este artículo con sus acertados comentarios. Asimismo, el autor quisiera extender su agradecimiento al ILS-Research Institute for Regional and Urban Development, Dortmund-Alemania, por la financiación económica recibida a través de un contrato de investigación post-doctoral en 2016. 


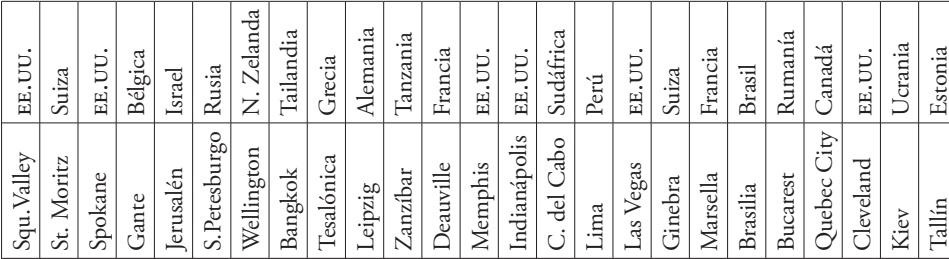

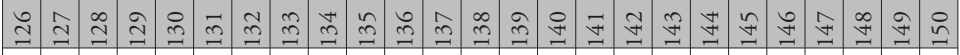

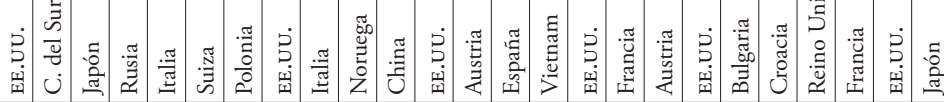

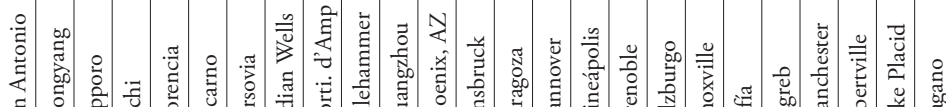

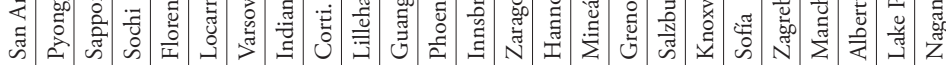

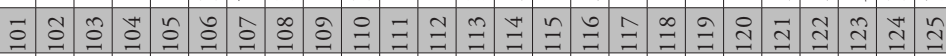

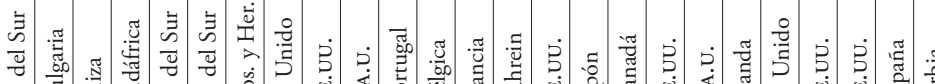

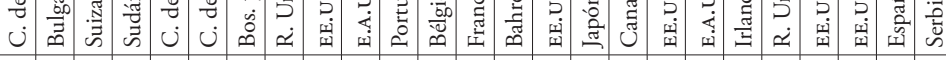

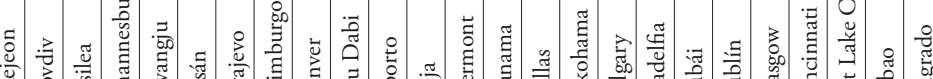

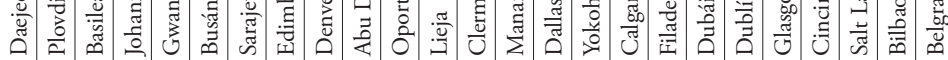

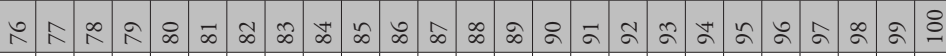

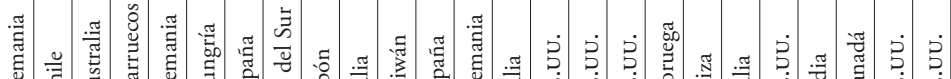

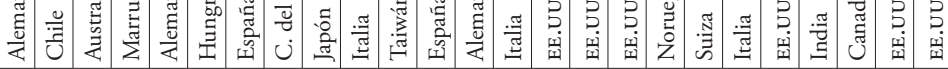

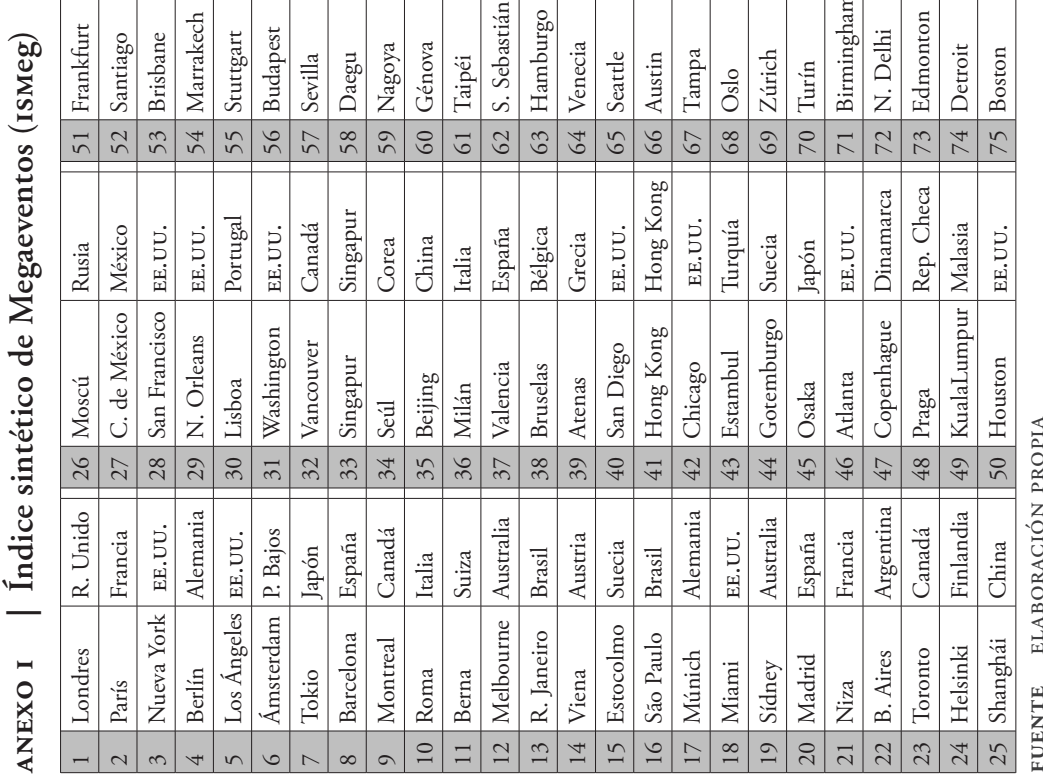


